\title{
Shock wave reflection from a wedge in a dusty gas
}

\author{
O. Igra ${ }^{\text {a }}{ }^{*}$, G. Hu ${ }^{\text {b }}$, J. Falcovitz ${ }^{\text {c }}$, B.Y. Wang ${ }^{\text {d }}$ \\ ${ }^{a}$ Department of Mechanical Engineering, Pearlstone Center for Aeronautical Engineering Studies, \\ Ben-Gurion University of the Negev, Beer Sheva, Israel \\ ${ }^{\mathrm{b}}$ Institute for Marine Dynamics, NRC, St. John's, Canada AlB $3 T 5$ \\ ${ }^{\mathrm{c}}$ Institute of Mathematics, Hebrew University of Jerusalem, Israel \\ ${ }^{\mathrm{d}}$ State Key Laboratory of Nonlinear Mechanics, Institute of Mechanics, Chinese Academy of Sciences, Beijing, China
}

Received 28 October 2003; received in revised form 29 April 2004

\begin{abstract}
The present paper contains a detailed study of shock wave reflection from a wedge placed in various suspensions. In past works, the incident shock propagated initially in pure gas and the suspension started only at the leading edge of the deflecting wedge. However, in the present case the entire flow field is filled with a gas-dust suspension and the initial shock wave has steady-state structure relative to the shock front. In former studies the transmitted shock wave starts its propagation into the suspension and is reflected from the wedge at the same time. It is therefore obvious that the two unrelated processes of (2D) reflection and (1D) "transitional" relaxation occur simultaneously. In the present case the suspension behind the incident shock wave has reached steady state (i.e., it is a traveling wave) before the shock reaches the wedge leading edge.

The reflection process from the deflecting wedge is studied for different dust mass loadings and different dust-particle diameter. It is shown that when the dust loading is low and the dust particle diameter is small the wave reflection pattern is similar to that observed in a similar pure gas case. In addition, an equilibrium state is reached, behind the evolved waves, very quickly. On the other hand, when the dust loading is relatively high and/or the dust particle diameter is relatively large, the observed reflection wave pattern is very different from that seen in a similar pure gas case. In such cases it takes much longer time to reach an equilibrium state behind the reflecting waves. It is also shown that the dust presence significantly affects the (gas) pressure on the wedge surface. The higher the dust loading is, the higher the pressure on the wedge surface. Suspensions composed of solid particle of different size, but having the same dust mass loading, will approach the same equilibrium pressure. However, it will take longer time to reach an equilibrium state for suspensions having large diameter particles.
\end{abstract}

(C) 2004 Elsevier Ltd. All rights reserved.

Keywords: Shock waves in suspensions; Shock wave reflection

${ }^{*}$ Corresponding author.

0301-9322/\$ - see front matter (C) 2004 Elsevier Ltd. All rights reserved.

doi:10.1016/j.ijmultiphaseflow.2004.05.008 


\section{Introduction}

Shock wave reflection from wedges has been thoroughly investigated, both experimentally and theoretically/numerically in the past four decades, and by now it is well understood and documented (e.g. in Ben-Dor, 1991). This is not the case when the compressible medium is a two-phase gas-dust suspension. In such cases experimental studies are extremely difficult to conduct, since it is almost impossible to produce a uniformly distributed dust in the suspension. (That is, small, spherical, rigid solid particles of equal diameter, which initially are uniformly distributed in the investigated suspension.) As a result, the few reported wedge-reflection studies that appeared in the archived literature are numerical studies of shock wave reflection from wedges placed in a suspension. Among such published studies one should mention the works of Kim and Chang (1991) and that of Ben-Dor et al. (2001).

The propagation of (planar) shock waves in suspensions was studied in the past four decades by many researchers, and by now it is well understood. At present a detailed description of shock wave propagation in suspensions can be found in books and review papers, for example in $\mathrm{Ru}-$ dinger's book (1980), in the review paper of Igra and Ben-Dor (1988) and in the recently published book of Crowe et al. (1998). It is shown in these references that when a shock wave travels through a gas-dust suspension (in equilibrium state), a relaxation zone follows the discontinuous jump in the flow properties of the gaseous phase that take place through the shock front. In the relaxation zone continuous changes in the suspension properties take place, via momentum and energy (heat) exchanges between the two phases. At the end of the relaxation zone a new state of equilibrium suspension is reached. The extent of the relaxation zone depends upon the solid particles diameter, the dust mass loading ratio in the suspension and the intensity of the incident shock wave.

The physical model used in the present paper is similar to that used in Kim and Chang (1991) and in Ben-Dor et al. (2001), i.e., it is based on the Euler equations for the gas and for a "zeropressure" dust continuum, plus the appropriate exchange terms. However, there is an essential difference between present and past numerical solutions. In the works of Kim and Chang (1991) and Ben-Dor et al. (2001) the incident shock propagates initially in a pure gas medium. The suspension starts only at the leading edge of the deflecting wedge. Therefore, the wave reflected from the wedge is the shock wave transmitted into the suspension as a result of the pure-air incident shock. This shock wave is weaker than the original incident shock wave in air. However, their results are shown as function of the original incident shock wave. Furthermore, since the transmitted shock wave starts its propagation into the suspension and is reflected from the wedge at the same time, it is obvious that the two unrelated processes of (2D) reflection and (1D) relaxation occur simultaneously. In other words, the "one-dimensional" interaction between the incident shock wave and the suspension, producing a reflected air shock and a transmitted shock propagating into the suspension takes place simultaneously with the two-dimensional shockwedge interaction. The one-dimensional transmitted shock evolves towards a "traveling wave" pattern of shock wave propagating in a suspension, where the leading "frozen" shock front is accompanied by a relaxation zone of finite extent. The overall shock-wedge interaction is consequently a superposition of the $1 \mathrm{D}$ evolution process and the $2 \mathrm{D}$ reflection. The resulting pattern is highly complex and difficult to interpret. Its only justification, in terms of research methodology, would be if it conformed to an existing experiment, or at least to a designed experiment. That 
is apparently not the case in the previously mentioned studies, and therefore we propose a different shock-suspension-wedge configuration.

In the proposed configuration the entire domain is filled with a stationary suspension. The incident shock wave propagates in the "traveling wave" mode well ahead of the wedge, and the width of the shock relaxation zone $\lambda$ is selected according to the following considerations. Let us denote by $L_{\mathrm{w}}$ the distance traveled by the incident shock front from $t=0$ when it arrives at the wedge leading edge, to some time $t>0$. We then propose to examine the interaction flow field at three different times. First where $L_{\mathrm{w}} \ll \lambda$, then at $L_{\mathrm{w}} \sim \lambda$ and last at $L_{\mathrm{w}} \gg \lambda$. At the early time we expect the $2 \mathrm{D}$ reflection pattern to be close to that of a pure gas shock of the same intensity as that of the frozen shock at the front of the incident wave. At the late time $\left(L_{\mathrm{w}} \gg \lambda\right)$ we might expect an almost pseudo-stationary pattern (this is suggested by reasoning that in the "thin shock" limit $\lambda=0$, the flow field will become self-similar, as in the pure gas case). In the middle case $\left(L_{\mathrm{w}} \approx \lambda\right)$ an intermediate pattern is expected. At any time, however, since the frozen shock at the front of the incident wave is of constant intensity, we should expect that near the reflection point on the wedge surface the reflection pattern will be close to the corresponding pure gas case (i.e., a pure gas shock of the same intensity as that of the leading frozen shock). This would be especially the case when the reflection is regular, or when it is a Mach reflection with a Mach stem shorter than $\lambda$. Another consideration, pertaining in particular to the case $L_{\mathrm{w}} \gg \lambda$, is that we can independently consider the reflection of an "equilibrium" incident shock wave. By that we mean the corresponding shock in an ideal gas having a polytropic coefficient $\gamma_{s}$ that represents full accommodation between the particles and the gas everywhere in the flow (Rudinger, 1980).

\section{Theoretical and numerical background}

In the present study a two-fluid model is employed for simulating the gas-particle suspension, where the solid phase is dilute enough to ignore its partial volume and its partial pressure in the suspension. This model is based on the "continuum assumption" for the solid (dust) phase, where the ensemble of particles is treated as a kind of "zero-pressure fluid" that interacts with the gas solely through an exchange of momentum and heat. The drag force and the rate of heat exchange are given in terms of single-particle empirical correlations involving the state of the gas near the particle. Assuming that the gas is inviscid, the gas flow is governed by the Euler equations expressing conservation of mass, momentum and energy. It is also assumed that the considered compressible gas can be treated as an ideal gas, i.e., it has a constant polytropic coefficient $\gamma$. Likewise, the "particle fluid" flow is also governed by the hydrodynamic conservation laws, but for a fluid having zero-pressure. Under these assumptions the governing equations for the considered nonstationary two-phase flow can be written in the Cartesian coordinates $(x, y)$ as:

$$
\begin{aligned}
& \frac{\partial U}{\partial t}+\frac{\partial E}{\partial x}+\frac{\partial F}{\partial y}=-S \quad \text { gas, } \\
& \frac{\partial V}{\partial t}+\frac{\partial G}{\partial x}+\frac{\partial H}{\partial y}=S \quad \text { particles }
\end{aligned}
$$


where the gas state and flux arrays are

$$
\begin{aligned}
& U(x, y, t)=\left(\begin{array}{c}
\rho_{\mathrm{g}} \\
\rho_{\mathrm{g}} u_{\mathrm{g}} \\
\rho_{\mathrm{g}} v_{\mathrm{g}} \\
\rho_{\mathrm{g}} E_{\mathrm{g}}
\end{array}\right) \quad E(U)=\left(\begin{array}{c}
\rho_{\mathrm{g}} u_{\mathrm{g}} \\
\rho_{\mathrm{g}} u_{\mathrm{g}}^{2}+p \\
\rho_{\mathrm{g}} u_{\mathrm{g}} v_{\mathrm{g}} \\
u_{\mathrm{g}}\left(\rho_{\mathrm{g}} E_{\mathrm{g}}+p\right)
\end{array}\right) \quad F(U)=\left(\begin{array}{c}
\rho_{\mathrm{g}} v_{\mathrm{g}} \\
\rho_{\mathrm{g}} u_{\mathrm{g}} v_{\mathrm{g}} \\
\rho_{\mathrm{g}} v_{\mathrm{g}}^{2}+p \\
v_{\mathrm{g}}\left(\rho_{\mathrm{g}} E_{\mathrm{g}}+p\right)
\end{array}\right) \\
& E_{\mathrm{g}}=e_{\mathrm{g}}+\frac{1}{2}\left(u_{\mathrm{g}}^{2}+v_{\mathrm{g}}^{2}\right) \quad p=\rho_{\mathrm{g}} R T_{\mathrm{g}}
\end{aligned}
$$

and the particles state and flux arrays are

$$
\begin{aligned}
& V(x, y, t)=\left(\begin{array}{c}
\rho_{\mathrm{p}} \\
\rho_{\mathrm{p}} u_{\mathrm{p}} \\
\rho_{\mathrm{p}} v_{\mathrm{p}} \\
\rho_{\mathrm{p}} E_{\mathrm{p}}
\end{array}\right) \quad G(V)=\left(\begin{array}{c}
\rho_{\mathrm{p}} u_{\mathrm{p}} \\
\rho_{\mathrm{p}} u_{\mathrm{p}}^{2} \\
\rho_{\mathrm{p}} u_{\mathrm{p}} v_{\mathrm{p}} \\
u_{\mathrm{p}} \rho_{\mathrm{p}} E_{\mathrm{p}}
\end{array}\right) \quad H(V)=\left(\begin{array}{c}
\rho_{\mathrm{p}} v_{\mathrm{p}} \\
\rho_{\mathrm{p}} u_{\mathrm{p}} v_{\mathrm{p}} \\
\rho_{\mathrm{p}} v_{\mathrm{p}}^{2} \\
v_{\mathrm{p}} \rho_{\mathrm{p}} E_{\mathrm{p}}
\end{array}\right) \\
& E_{\mathrm{p}}=C_{m} T_{\mathrm{p}}+\frac{1}{2}\left(u_{\mathrm{p}}^{2}+v_{\mathrm{p}}^{2}\right)
\end{aligned}
$$

The source array is

$$
S(U, V)=\left(\begin{array}{c}
0 \\
f_{x} \\
f_{y} \\
u_{\mathrm{p}} f_{x}+v_{\mathrm{p}} f_{y}+q
\end{array}\right)
$$

In Eqs. (1)-(7) variables $p, \rho, T$ and $u, v$ stand for pressure, density, temperature and velocity components in the $x$ - and $y$-directions, respectively. Subscripts $\mathrm{g}$ and $\mathrm{p}$ refer to the gas and the solid particles, respectively. The gas pressure is expressed in terms of the other gas properties, i.e., $p=(\gamma-1) \rho e_{\mathrm{g}} ; e_{\mathrm{g}}$ is the gas specific energy. $C_{m}$ is the specific heat capacity of the solid phase. The source terms appearing on the right hand side of Eqs. (1) and (2) represent the coupling via momentum and energy exchange between the two phases. The viscous interaction between the gas and the solid phase is accounted for by employing the following drag force $\left(f_{x}, f_{y}\right)$, and a heat exchange rate $q$ expressions:

$$
\begin{aligned}
& f_{x}=\frac{\pi D_{\mathrm{p}}^{2}}{8 m} \rho_{\mathrm{p}} \rho_{\mathrm{g}}\left(u_{\mathrm{g}}-u_{\mathrm{p}}\right) \sqrt{\left(u_{\mathrm{g}}-u_{\mathrm{p}}\right)^{2}+\left(v_{\mathrm{g}}-v_{\mathrm{p}}\right)^{2}} C_{\mathrm{D}} \\
& f_{y}=\frac{\pi D_{\mathrm{p}}^{2}}{8 m} \rho_{\mathrm{p}} \rho_{\mathrm{g}}\left(v_{\mathrm{g}}-v_{\mathrm{p}}\right) \sqrt{\left(u_{\mathrm{g}}-u_{\mathrm{p}}\right)^{2}+\left(v_{\mathrm{g}}-v_{\mathrm{p}}\right)^{2}} C_{\mathrm{D}} \\
& q=\frac{\pi D_{\mathrm{p}}}{m} \cdot \rho_{\mathrm{p}} \frac{\mu C_{\mathrm{p}}}{P r}\left(T_{\mathrm{g}}-T_{\mathrm{p}}\right) N u
\end{aligned}
$$

where $m$ and $D_{\mathrm{p}}$ are the mass and diameter of a single solid particle; $\mu, \operatorname{Pr}$ and $C_{p}$ are the gas viscosity, Prandtl number and specific heat capacity at constant pressure, respectively. In addition, empirical expressions for the drag coefficient $C_{\mathrm{D}}$ and the Nusselt number $N u$ are chosen from the literature. Frequently used expressions are (Igra and Ben-Dor, 1988):

$$
C_{\mathrm{D}}=24 R e^{-1}+4 R e_{\mathrm{s}}^{-1 / 3}
$$




$$
\begin{aligned}
& N u=2.0+0.6 P^{\frac{1}{3}} R e_{\mathrm{s}}^{\frac{1}{2}} \\
& R e_{\mathrm{s}}=\rho_{\mathrm{g}}\left[\left(u_{\mathrm{g}}-u_{\mathrm{p}}\right)^{2}+\left(v_{\mathrm{g}}-v_{\mathrm{p}}\right)^{2}\right]^{1 / 2} D_{\mathrm{p}} / \mu
\end{aligned}
$$

where $R e_{\mathrm{s}}$ is the slip Reynolds number based on the particle diameter. Here we observe a nearly complete analogy in the conservation laws for the gas and particle phase. The only difference in the conservation laws stems from the absence of pressure in the solid phase (and hence the absence of an "equation of state").

It is questionable, however, whether the total energy conservation law for the particles is physically appropriate. Under this law, the particles kinetic energy may be converted into thermal energy without resorting to the gas as an "intermediary" medium. This situation may be remedied by replacing the total energy equation by the thermal energy conservation law,

$$
\partial_{t}\left(\rho_{\mathrm{p}} C_{m} T_{\mathrm{p}}\right)+\partial_{x}\left(\rho_{\mathrm{p}} u_{\mathrm{p}} C_{m} T_{\mathrm{p}}\right)+\partial_{y}\left(\rho_{\mathrm{p}} v_{\mathrm{p}} C_{m} T_{\mathrm{p}}\right)=q
$$

which is derived by combining the particle mass and momentum equations with the total energy conservation law. It is noted that unlike the respective equation for a compressible fluid, the particle "internal energy" (Eq. (8)) retains its conservation law form due to the absence of pressure. In the present study we adopt this "thermal energy" conservation law for the particle, and defer the issue of comparing the two options to a future study.

Using the operator-splitting technique, Eq. (1) is replaced by the following two conservation laws:

$$
\begin{aligned}
& \frac{\partial U}{\partial t}+\frac{\partial E}{\partial x}=-\frac{1}{2} S \\
& \frac{\partial U}{\partial t}+\frac{\partial F}{\partial y}=-\frac{1}{2} S
\end{aligned}
$$

An analogous splitting is performed on the particles Eq. (2).

Due to the split procedure, the two-dimensional finite-difference scheme for the integration of Eq. (1) can be obtained by applying a sequence of one-dimensional conservation law schemes to Eq. (9), and the respective particles phase equations. The method used here is the secondorder accurate GRP scheme. We refer the reader to Falcovitz and Ben-Artzi (1995) for an extensive review of the GRP principles and its fluid dynamical implementations. In the following, the one-dimensional GRP scheme used for solving Eq. (9a) is briefly outlined. Let the computational domain be divided into a grid of equally spaced points $x_{i+1 / 2}=$ $(i+1 / 2) \Delta x$ where $\Delta x$ is the grid spacing and the $i$ th cell is the interval $x_{i-1 / 2}<x<x_{i+1 / 2}$. The conservative second-order difference scheme for time integration of the conservation laws, (Eq. (9a)), is

$$
\begin{aligned}
& U_{i}^{n+1}=U_{i}^{n}-\frac{\Delta t}{\Delta x}\left[E(U)_{i+1 / 2}^{n+1 / 2}-E(U)_{i-1 / 2}^{n+1 / 2}\right]-\frac{\Delta t}{2} S_{i}^{n}, \\
& \Delta t=t_{n+1}-t_{n} .
\end{aligned}
$$

where $U_{i}^{n}$ denotes the average value of $U$ in cell $i$ at time $t=t_{n}=n \Delta t$, and where the time-centered fluxes $E(U)_{i+1 / 2}^{n+1 / 2}$ are obtained analytically from solutions to generalized Riemann problems that 
arise at cell interfaces $x_{i+1 / 2}$ as a result of the piecewise linear approximation to $U\left(x, y, t_{n}\right)$ in cell $i$. The unique feature of the GRP scheme is that those fluxes are calculated from closed-form expressions obtained as an analytic solution to each generalized Riemann problem.

As for the particles integration scheme, U, E, F, $-\mathrm{S}$ are replaced by $\mathrm{V}, \mathrm{G}, \mathrm{H}, \mathrm{S}$. There is a significant change, however, in regards to the Riemann problem and the generalized Riemann problem at cell-interfaces. Due to the absence of pressure, the particles conservation laws admit only a contact-type discontinuous solution, with a possible jump in particles density and temperature, but continuous velocity. The Riemann problem at $x_{i+1 / 2}$ thus assumes a particularly simple solution: the flow variables at $t>t_{n}$ are taken from the distribution in the "upwind" adjacent cell, according to the sign of average particles velocity on either side of $x_{i+1 / 2}$ (thereby disregarding the "forbidden" jump in velocity).

Two different suspensions were treated in the present work. One had a relatively high dust mass loading, $\eta=1$, while the other had a relatively low dust mass loading, $\eta=0.1$ ( $\eta$ is the dust-gas mass ratio). It should be mentioned that even when $\eta=1$ the number of solid particles present in the suspension is very small, and the distance between neighboring solid particles is relatively large, due to the very large difference in mass between the two components (phases) of the suspension.

Assuming a steady shock wave in a gas-dust suspension, its structure is governed by the time-independent conservation laws expressed in a coordinate system attached to the traveling shock wave. This set of ordinary differential equations is readily integrated using standard methods, such as the Runge-Kutta method. Steady shock in dusty suspension has been presented in past publications, such as the review by Igra and Ben-Dor (1988). In the present study we use a piston moving at constant velocity for generating a planar shock wave propagating into a uniform quiescent gas-particles suspensions (Falcovitz and Igra, 2000). The piston speed ${ }^{1}$ is set to 1.0 for dust mass loading of $\eta=1$ and to 0.64 for $\eta=0.1$. These two speeds generate the same frozen pressure jump in the leading air shock wave, $p / p_{1}=2.069$, independently of the particles diameter. However, the relaxation length varies for the different particles diameter.

In the present work the computational domain was a rectangle of $346 \times 95 \mathrm{~mm}$ for suspensions composed of particles whose diameter was $d=1,5$, or $10 \mu \mathrm{m}$. It was a rectangle of $600 \times 95 \mathrm{~mm}$ for the suspension in which the particle's diameter was $d=20 \mu \mathrm{m}$. The investigated wedge is 136 $\mathrm{mm}$ long and it has a deflecting angle of $\theta_{\mathrm{w}}=30.5^{\circ}$. The initial conditions were set as follows: The incident (frozen) shock front was positioned $20 \mathrm{~mm}$ ahead of the wedge, so that the GRP computation would compute the propagation of the shock through that distance, prior to the beginning of its interaction with the wedge. Ahead of the shock front the suspension conditions were specified $\left(T_{\mathrm{g}}=T_{\mathrm{p}}=294 \mathrm{~K}, p=1\right.$ bar, $\rho_{\mathrm{g}}=1.22 \mathrm{~kg} / \mathrm{m}^{3}, \eta=0.1$ or 1$)$. Downstream of the front, we specified the suspension profile obtained from the previously cited calculation of the steady shock structure. The length of the computational domain was such that post-shock equilibrium was reached well within the domain.

\footnotetext{
${ }^{1}$ Note that the piston speed is equal to the equilibrium post-shock velocity of the suspension. As in the pure gas case, this single variable determines a unique (steady) shock wave.
} 
The wedge boundary is implemented in the two-dimensional space by treating it as a straight line that cuts a regular Cartesian grid. We apply a moving boundary tracking (MBT) scheme to the irregular cells formed by the intersection of the wedge with the regular mesh, as described in Falcovtiz et al. (1997). In general, the boundary line may be moving, but here it is stationary. The boundary condition for the particle flux at the oblique (wedge) line is zero mass and energy fluxes, and zero flux for the normal component of the momentum flux. The tangential momentum flux component is also set to zero, which implies that the tangential particle velocity is unchanged by the oblique boundary (in other words, particles are specularly reflected).

\section{Results and discussion}

The previously described GRP scheme was successfully employed for solving complex shock wave interactions in pure gas (see for example Igra et al., 1996, 2001, Falcovtiz et al., 1993 and Falcovitz and Ben-Artzi, 1995). In these papers the numerical solutions were compared with experimental findings and excellent agreement was found between the two, confirming the reliability of the numerical solution obtained for the considered cases. In the present case the flow takes place in a two-phase medium. Unfortunately, to the best of our knowledge there are no experimental results that can be used as a bench mark for the presently considered flows. Therefore, we started the present study by repeating Kim and Chang (1991) work using the GRP scheme. The obtained results are shown in Fig. 1. It is clear from this figure that very good agreement is found between the two sets of numerical results obtained by using different numerical schemes. In Kim and Chang (1991) results all shock waves are smeared over a few grid points. They are clearly and sharply seen in the present results.

As mentioned earlier, in the present solutions the incident shock wave propagates in a suspension throughout the investigated flow field; there is no initial 'pure gas' zone in the results shown subsequently. The solution is conducted for the following initial conditions: The frozen pressure jump across the traveling shock wave is $P / P_{0}=2.069$ (which corresponds to $\left.M_{\mathrm{s}}=1.3843\right), P_{0}=1$ bar, $\rho_{\mathrm{g} 0}=1.22 \mathrm{~kg} / \mathrm{m}^{3}$ and $T_{0}=294 \mathrm{~K}$. The wedge deflection angle is $30.5^{\circ}$; solutions were sought for dust (mass) loading ratios of $\eta=0.1$ and 1.0 and dust particle diameter of, $D_{\mathrm{p}}=1,5,10$ and $20 \mu \mathrm{m}$. The solid dust particles have material density of $2500 \mathrm{~kg} / \mathrm{m}^{3}$ and the specific heat ratio, air-solid, $\delta=C_{m} / C_{\mathrm{p}}=1$. For the gaseous phase (air) we used $\gamma=1.4$ and molecular weight of 29. The initial flow profile behind the incident shock wave was obtained as follows. We integrated the ordinary differential equations for the steady flow behind a stationary shock front in a dusty suspension, using the same two-phase dusty model described earlier. This solution was transformed to a "traveling wave" frame, simply by adding a constant velocity such that the suspension ahead of the shock became stationary. The case $\eta=1, D_{\mathrm{p}}=5 \mu \mathrm{m}$ is shown in Fig. 2, at the moment $(t=0)$ where the shock front is positioned at $x=0.180 \mathrm{~m}$. In Fig. 2 profiles of the gas pressure $P$, gas velocity $u_{\mathrm{g}}$ and the dust particle velocity $u_{\mathrm{p}}$ are shown. A jump in $P$ and $u_{\mathrm{g}}$ at the ("frozen") shock front is clearly visible. There is no jump in $u_{\mathrm{p}}$. The continuity in $u_{\mathrm{p}}$ is evidence of the finite (mass) inertia of the dust particles. A mechanical equilibrium, i.e., $u_{\mathrm{p}}=u_{\mathrm{g}}$ is reached after a relaxation distance of about $0.08 \mathrm{~m}$ (at $x \approx 0.1 \mathrm{~m}$ in Fig. 2). A similar pattern is observed when checking the gas density $\rho_{\mathrm{g}}$ and temperature $T_{\mathrm{g}}$, and the dust temperature $T_{\mathrm{p}}$. We 

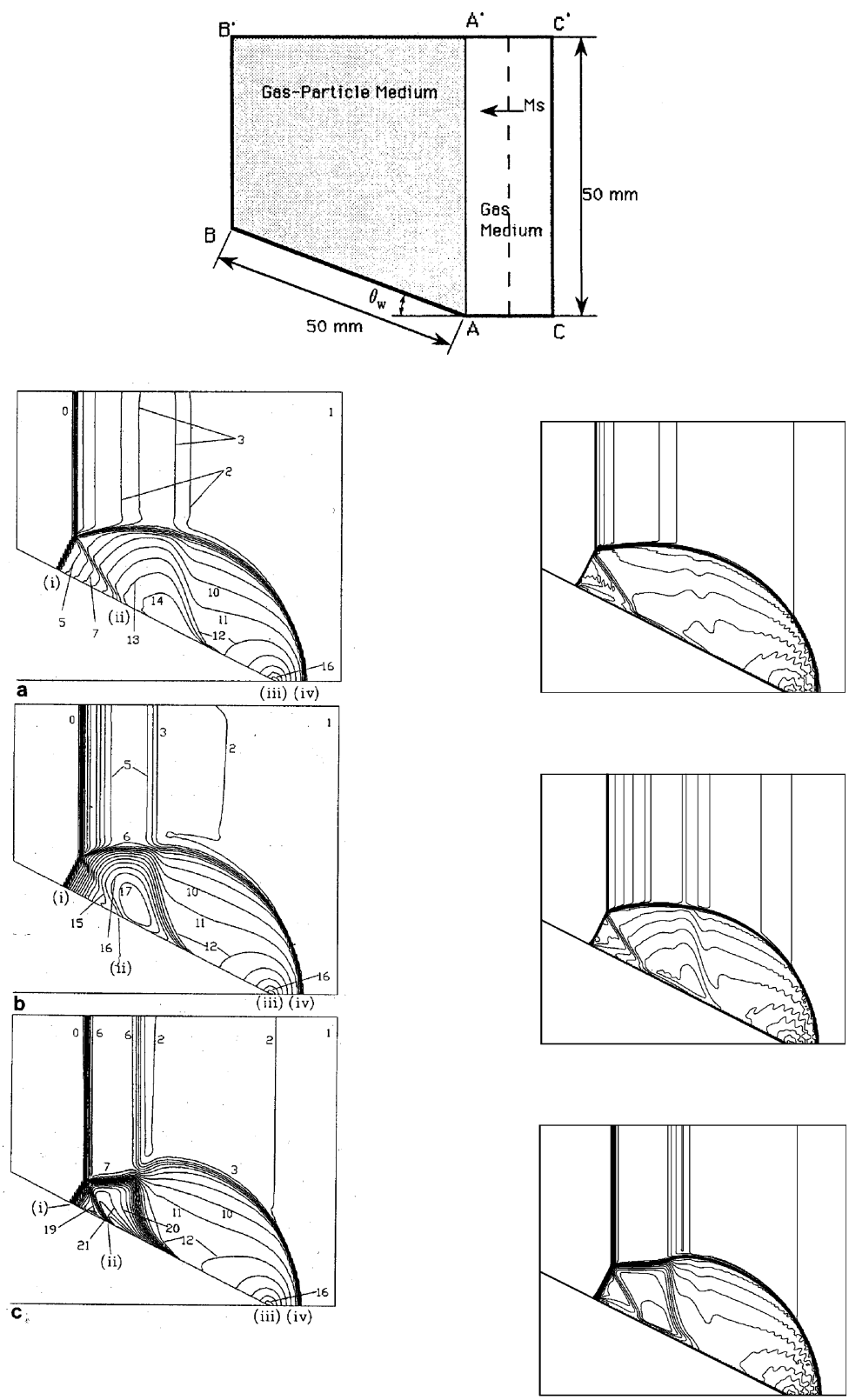

Kim \& Chang's results

Present results

Fig. 1. Comparison between Kim and Chang's results and present computations. $M_{\mathrm{s}}=2.03, \theta_{\mathrm{w}}=27^{\circ}$ and the dust loading is equal to 0.23 . (a) $D_{\mathrm{p}}=10 \mu \mathrm{m}$, (b) $D_{\mathrm{p}}=5 \mu \mathrm{m}$, and (c) $D_{\mathrm{p}}=1 \mu \mathrm{m}$.

have also obtained the respective 1D profiles for all other cases, but we do not show them graphically here. The estimated relaxation lengths for the various particles diameter (and $\eta=1$ ) 


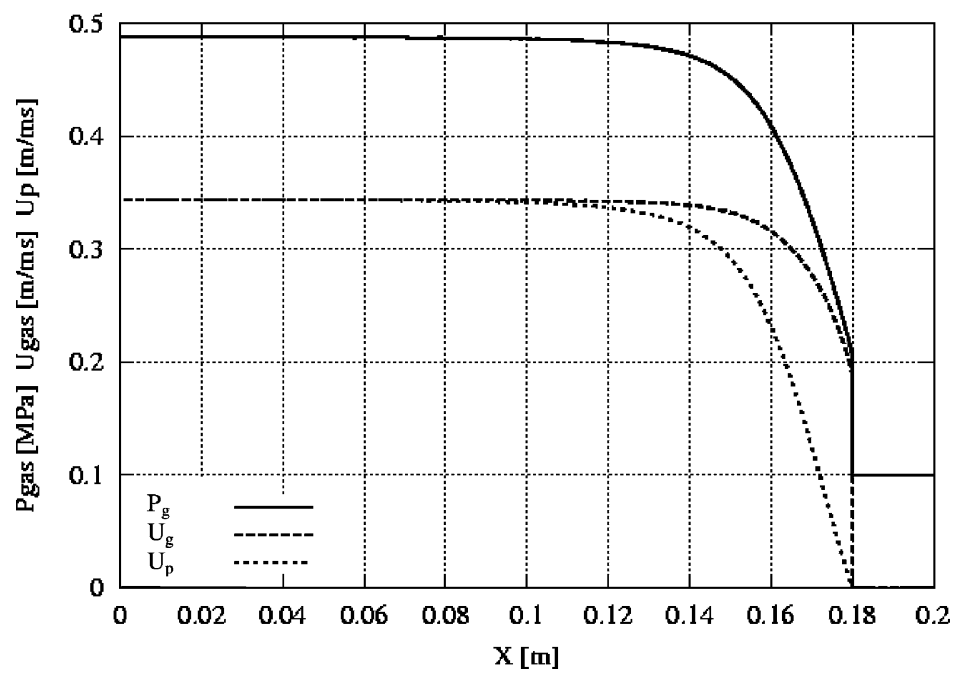

Fig. 2. Steady shock wave in dusty gas. $D_{\mathrm{p}}=5 \mu \mathrm{m}, \eta=1, P_{0}=1$ bar and $T_{0}=294 \mathrm{~K}$.

Table 1

Relaxation length $\lambda$ for various particles diameters $D_{\mathrm{p}}$

\begin{tabular}{lll}
\hline$D_{\mathrm{p}}(\mu \mathrm{m})$ & (a) $\eta=1, \lambda(\mathrm{m})$ & (b) $\eta=0.1, \lambda(\mathrm{m})$ \\
\hline 1 & 0.005 & 0.008 \\
5 & 0.080 & 0.140 \\
10 & 0.200 & 0.500 \\
20 & 0.600 & 1.600 \\
\hline
\end{tabular}

Leading shock in air $M_{\mathrm{s}}=1.384$.

are summarized in Table 1 (column a). For the lower dust loading, the relaxation lengths are higher, as summarized in column $b$ of Table 1 .

The obtained results are shown subsequently in four different subsections. First, results obtained for the gaseous phase of the suspension are discussed; this is followed by presentation of results obtained for the solid phase (the dust cloud). Thereafter, results obtained for equilibrium suspension (pseudo-gas) are given, and the last to be discussed is the pressure distribution along the wedge surface for various suspensions.

\subsection{Flow maps of the gas phase}

Obtained results, showing lines of constant density (isopycnics) in the gaseous phase, are shown in Figs. 3-12. In Figs. 3-5 results obtained for the smallest particles $\left(D_{\mathrm{p}}=1 \mu \mathrm{m}\right)$ are presented. Referring to Table 1, the width of the relaxation zone (which in Fig. $3 \mathrm{~b}$ and c looks like a bold line) indicates that the present case falls into the category of $L_{\mathrm{w}} \gg \lambda$ where $L_{\mathrm{w}}$ is taken as the 


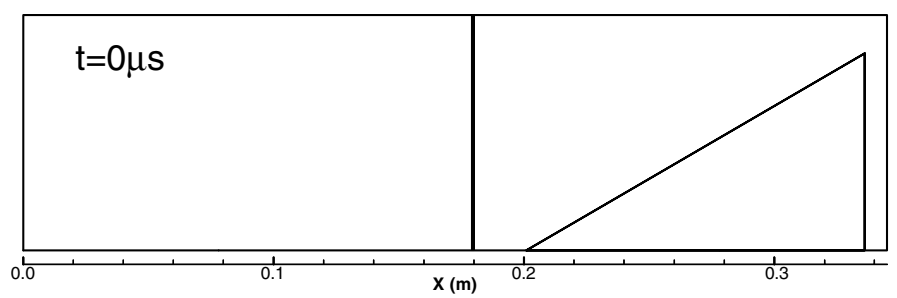

(a)

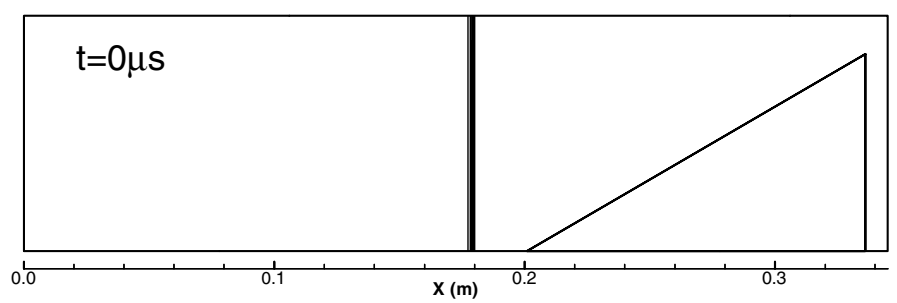

(b)

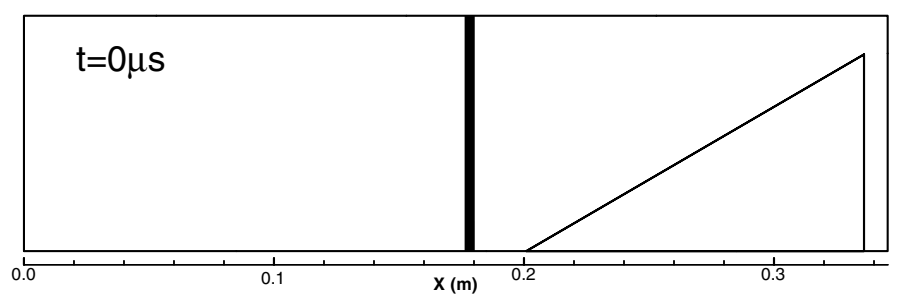

(c)

Fig. 3. Shock wave reflection from a wedge placed inside a suspension. $M_{\mathrm{s}}=1.384, P_{0}=1$ bar, $T_{0}=294 \mathrm{~K}$ and $D_{\mathrm{p}}=1$ $\mu \mathrm{m}$ : (a) pure gas, (b) suspension having a dust loading equal 0.1 and (c) dust loading equal $1.0, t=0 \mu \mathrm{s}$.

wedge length. With progressing time the shock reflection from the wedge takes place, the situation at $t=200 \mu \mathrm{s}$ is presented in Fig. 4. As could be expected, when the particles diameter is very small $\left(D_{\mathrm{p}}=1 \mu \mathrm{m}\right)$ and the dust loading is also low $(\eta=0.1)$, the solid phase in the suspension responds quickly to abrupt changes in the gaseous phase taking place behind the incident and the reflected shock waves. As a result, similar wave patterns are shown in Fig. 4a and b. In Fig. 4c the dust loading is 10 times higher than in $4 \mathrm{~b}$ and this affects significantly the transition to equilibrium in the post-shock suspension as is evident from Fig. 4c. In Fig. 4c the reflected shock wave from the wedge degenerated to a compression wave. It is worth noting that all shock waves shown in Fig. 4 reached, or almost reached, equilibrium within the computed flow duration and the observed wave geometry is similar to that obtained in a quasi-steady flow. Later on, in Fig. 18, it will be shown that the obtained wave pattern is similar to that observed while treating the suspension as an equilibrium-state pure gas. Since the frozen shock at the front of the incident wave is of constant intensity, the reflection pattern close to the reflection point is almost the same in Fig. 4ac. Only further away from the reflection point a difference is noticed. The latest result is presented in Fig. 5 showing the flow field at $t=300 \mu \mathrm{s}$. It is clear here that while the results obtained for 


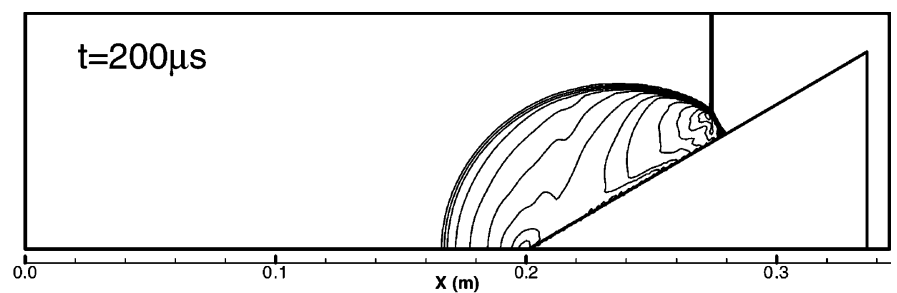

(a)

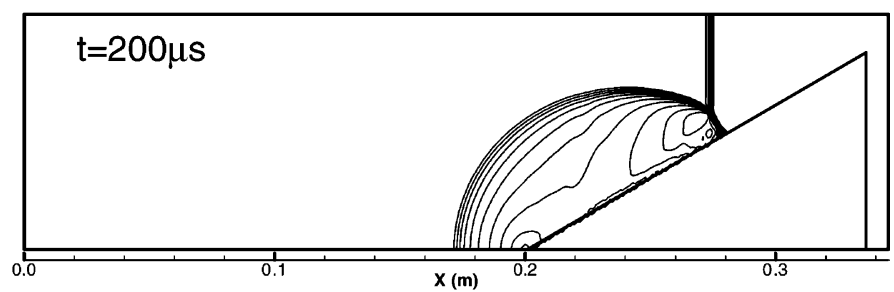

(b)

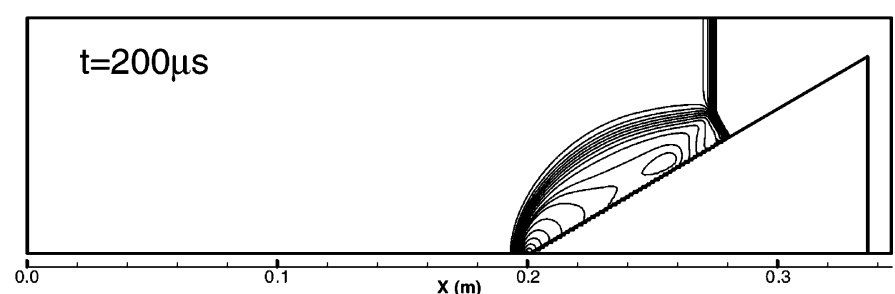

(c)

Fig. 4. Shock wave reflection from a wedge placed inside a suspension. $M_{\mathrm{s}}=1.384, P_{0}=1$ bar, $T_{0}=294 \mathrm{~K}$ and $D_{\mathrm{p}}=1$ $\mu \mathrm{m}$ : (a) pure gas, (b) suspension having a dust loading equal 0.1 and (c) dust loading equal 1.0, $t=200 \mu \mathrm{s}$.

$\eta=0.1$ (Fig. 5b) are similar to those obtained for a pure gas case (Fig. 5a), those obtained for $\eta=1$ (Fig. 5c) are very different. The massive dust presence $(\eta=1)$ altered the reflection process; now it is clearly evident that the reflected shock from the wedge is reduced to a compression wave and the shock reflected from the conduit's ceiling (which is clearly visible in Fig. 5a and b) decayed to Mach waves and is not visible in Fig. 5c.

Results obtained for larger solid particles, $D_{\mathrm{p}}=5 \mu \mathrm{m}$, are shown in Figs. 6-8. In Fig. 6 the flow field is shown shortly before the traveling shock wave has reached the wedge leading edge. As expected, when the particles diameter in the suspension is enlarged, the extent of the relaxation zone is also enlarged; this is evident from comparing Fig. 6b and/or c with Fig. 3b and/or c (see also Table 1). The present case falls in the category $L_{\mathrm{w}} \sim \lambda$, where $L_{\mathrm{w}}$ is taken as the wedge length. Enlarging the solid particles diameter affects the entire suspension flow and not only the extent of the relaxation zone developed behind the traveling shock wave, especially in the case of high dust loading ratio. Comparing Figs. 7 and 8 with 4 and 5 shows that the wave pattern of the reflection process is changed. While in Figs. 4 and 5 the expected geometry of Mach reflection is evident (although in Fig. 5c the reflected shock wave has decayed to a compression wave), in Figs. 7c and $8 \mathrm{c}$ the reflected shock is fully dispersed and the entire flow behind the traveling shock and its 


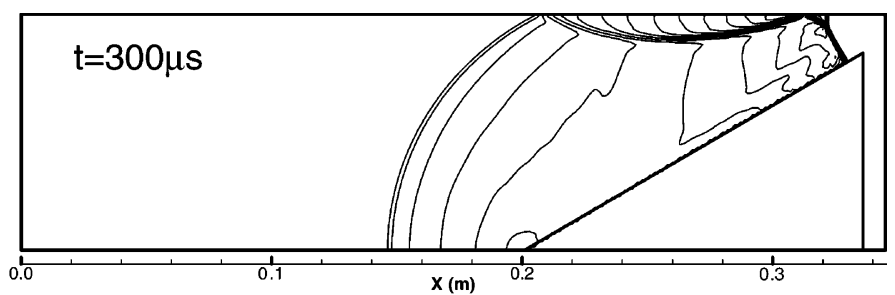

(a)

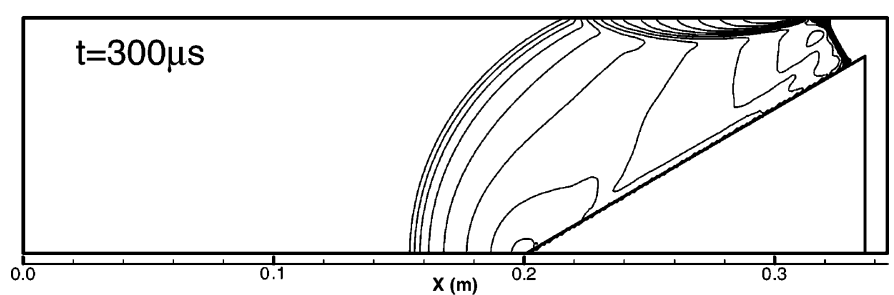

(b)

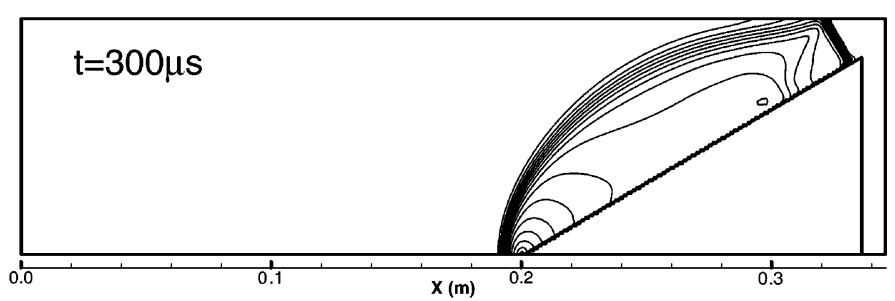

(c)

Fig. 5. Shock wave reflection from a wedge placed inside a suspension. $M_{\mathrm{s}}=1.384, P_{0}=1$ bar, $T_{0}=294 \mathrm{~K}$ and $D_{\mathrm{p}}=1$ $\mu \mathrm{m}$ : (a) pure gas, (b) suspension having a dust loading equal 0.1 and (c) dust loading equal $1.0, t=300 \mu \mathrm{s}$.

Mach stem is smooth, i.e., it is a compression wave. The wave pattern observed in Fig. 8c is different from the similar pure gas case shown in Fig. 8a. A further increase of the solid particles diameter, in the suspension, causes even greater departure from a similar pure gas case.

In Figs 9 and 10 results obtained for $D_{\mathrm{p}}=10 \mu \mathrm{m}$ are shown. It is apparent from these figures that the length of the relaxation zone developed behind the traveling shock is further extended; it covers now more than $10 \mathrm{~cm}$ (see Fig. $9 \mathrm{~b}$ and c (see also Table 1)). This means that in the present case we are still within the domain where $L_{\mathrm{w}} \approx \lambda$. As observed for the $5 \mu \mathrm{m}$ particles here too, in the high dust loading case (Fig. 10c) the reflected shocks, from the wedge and from the duct ceiling, are reduced to compression waves, which is very different from the Mach reflection pattern observed in a similar pure gas case (Fig. 10a) or in the case of a dilute suspension (Fig. 10b). In Fig. 10c one sees a shift in the isopycnic lines, behind the Mach stem, close to the wedge surface. As will be shown later this shift is due to large dust accumulation close to the wedge surface.

In the last case to be discussed, shown in Figs. 11 and 12, the solid particles diameter is increased to $20 \mu \mathrm{m}$. This increase in the particles diameter results in extending the relaxation zone developed behind the incident shock wave to $0.600 \mathrm{~m}$ (see Table 1). Since the pure gas case is identical to that shown in previous figures it is not repeated in Figs. 11 and 12. Now the 


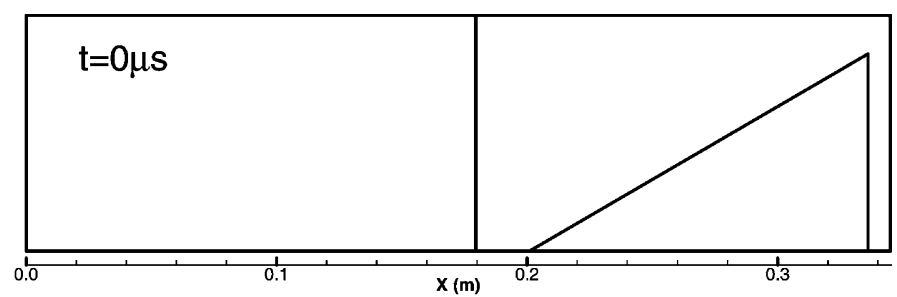

(a)

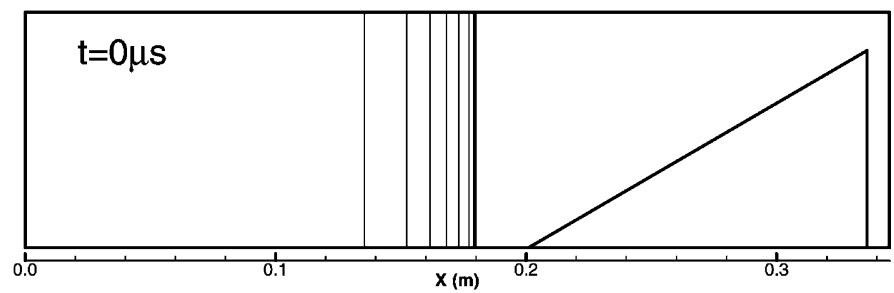

(b)

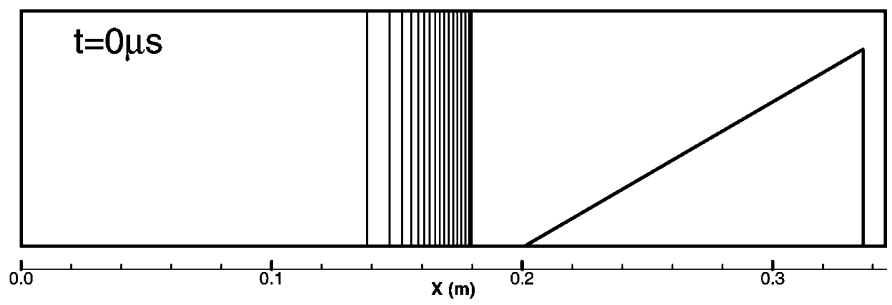

(c)

Fig. 6. Shock wave reflection from a wedge placed inside a suspension. $M_{\mathrm{s}}=1.384, P_{0}=1$ bar, $T_{0}=294 \mathrm{~K}$ and $D_{\mathrm{p}}=5$ $\mu \mathrm{m}$ : (a) pure gas, (b) suspension having a dust loading equal 0.1 and (c) dust loading equal $1.0, t=0 \mu \mathrm{s}$.

relaxation zone is significantly larger than the wedge length $\left(L_{\mathrm{w}} \ll \lambda\right)$ and the reflection process shown in Fig. 12 is in a nonequilibrium state. It is impossible to speak about a quasi-steady state in the present case since here we are still within the relaxation process. (This will be clearly demonstrated later when pressure distribution along the wedge surface is discussed.) As could be expected for a case where $L_{\mathrm{w}} \ll \lambda$, at early times the reflection wave pattern is similar to that of the pure gas case. This indeed is the case as is evident from Fig. 12 where one still observes the reflected shock wave from the duct ceiling even when $\eta=1$, a phenomenon not observed in Fig. 8c or in Fig. 10c.

\subsection{Flow maps of the particles phase}

In Figs. 3-12 the flow evolved behind the traveling shock wave during its reflection from the wedge was shown via maps of isopycnics in the gaseous phase of the suspension. To complete the picture isopycnics in the solid phase are shown in Figs. 13-16. In Fig. 13 results obtained for 


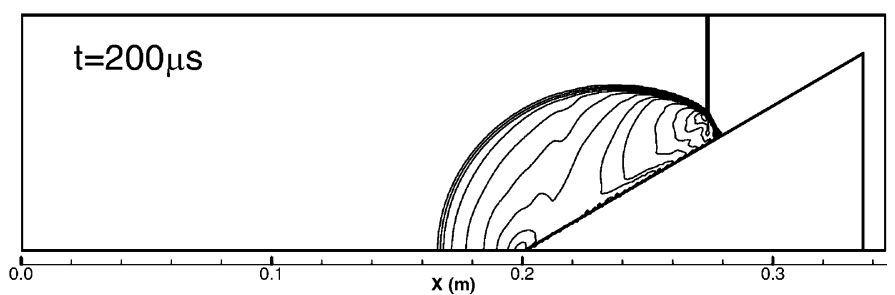

(a)

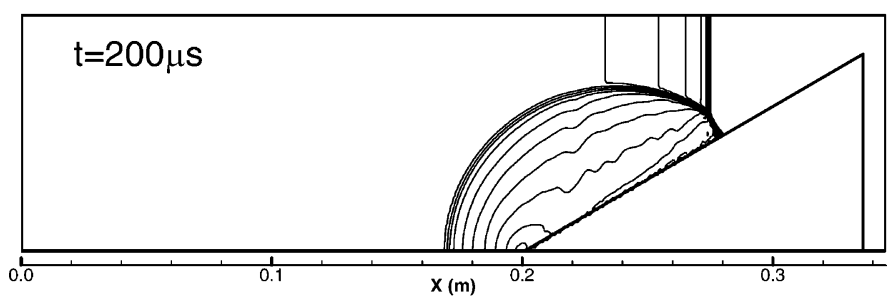

(b)

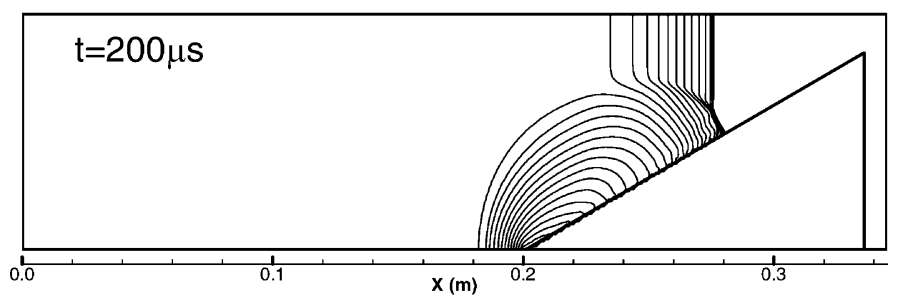

(c)

Fig. 7. Shock wave reflection from a wedge placed inside a suspension. $M_{\mathrm{s}}=1.384, P_{0}=1$ bar, $T_{0}=294 \mathrm{~K}$ and $D_{\mathrm{p}}=5$ $\mu \mathrm{m}$ : (a) pure gas, (b) suspension having a dust loading equal 0.1 and (c) dust loading equal $1.0, t=200 \mu \mathrm{s}$.

the small particles $\left(D_{\mathrm{p}}=1 \mu \mathrm{m}\right)$ and low dust loading ratio $(\eta=0.1)$ are shown. Comparing results shown in Fig. 13 with those of Figs. $4 \mathrm{~b}$ and $5 \mathrm{~b}$ indicate that there is some similarity between wave patterns shown in the two sets. This is expected since for such small particles and such minute amount of dust the response to abrupt changes is very quick (due to small inertia of the considered particles), and in regions of large gradients in the gaseous phase one sees large dust gradients as well. Therefore, in Fig. 13 shock and/or compression waves (in the gaseous phase) are clearly visible in the solid phase isopycnics; they are similar in shape to the corresponding isopycnics map in the gaseous phase. However, while for $t=200 \mu \mathrm{s}$ (Fig. 4b) in the gaseous phase the reflected shock wave is clearly visible, first as a shock and away from the triple point as an intense compression wave, in Fig. 13 (for $t=200 \mu$ s) it appears as a weak compression/Mach wave. The difference between the gaseous phase and the solid phase isopycnics becomes more pronounced at the later time, at $t=300 \mu \mathrm{s}$. In Fig. $5 \mathrm{~b}$ a reflected shock wave/compression wave from the conduit ceiling is clearly observed. In Fig. 13 (for $t=300 \mu \mathrm{s}$ ) the reflection from the conduit ceiling is hardly visible; it is shown as a gradual change in density, i.e., a compression wave. When the particle's diameter is increased, its inertia is also increased proportionately to $D_{\mathrm{p}}^{3}$. This will alter the "wave" pattern in the solid phase of the suspension as is evident from comparing Fig. 14 with 


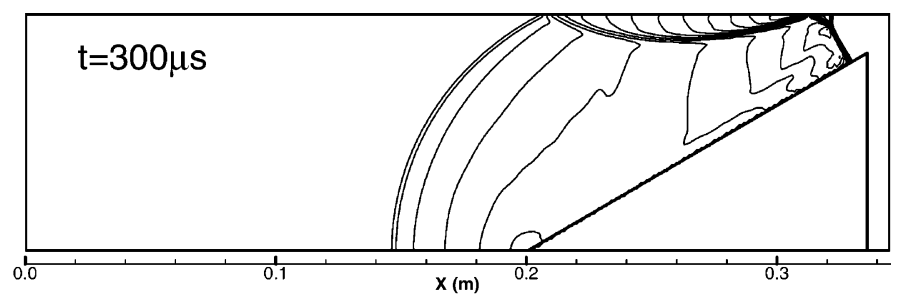

(a)

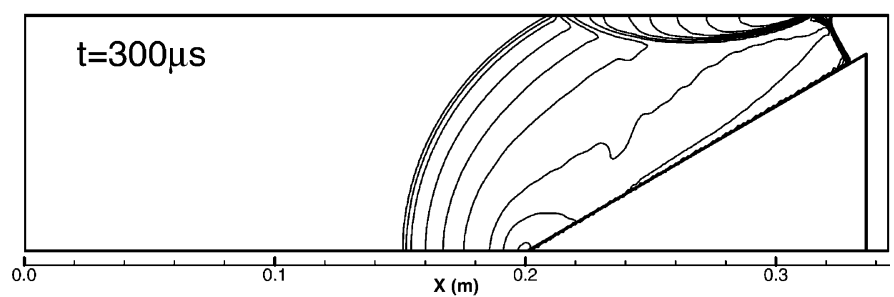

(b)

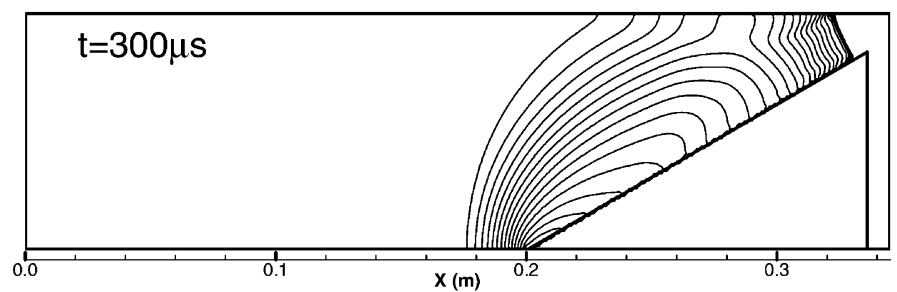

(c)

Fig. 8. Shock wave reflection from a wedge placed inside a suspension. $M_{\mathrm{s}}=1.384, P_{0}=1$ bar, $T_{0}=294 \mathrm{~K}$ and $D_{\mathrm{p}}=5$ $\mu \mathrm{m}$ : (a) pure gas, (b) suspension having a dust loading equal 0.1 and (c) dust loading equal $1.0, t=300 \mu \mathrm{s}$.

Figs. $7 \mathrm{~b}$ and $8 \mathrm{~b}$. The isopycnics observed in the solid phase bear no similarity to those observed in the gaseous phase. The discrepancy between the two will remain or will increase as the diameter of the solid particles in the suspension increases. Also, the incident shock wave, which is preceded by a distinct front in Figs. 7b and 8b, is reduced to a band of isopycnic lines in Fig. 14 (corresponding to the smooth variation of particles phase density behind the incident shock front). What is shown clearly in Fig. 14 and is not visible in Figs. 7-9, 11, 12 is the high dust (solid particles) concentration near the wedge surface. This high dust concentration near the wedge surface is persistent for all particles with diameter equal or larger than $5 \mu \mathrm{m}$. It is an accumulation of particles on the surface, due to the no-rebound boundary condition there.

Increasing the dust concentration in the suspension (specifically, increasing $\eta$ tenfold from 0.1 to 1) enhances the previously mentioned phenomenon. For the case of small dust particles (Fig. 15) the observed wave pattern is similar to that seen in the appropriate gaseous phase isopycnics (Figs. $4 \mathrm{c}$ and $5 \mathrm{c}$ ). In both cases the isopycnics suggest that an equilibrium state is reached (see Figs. 13 and 15), as could be expected for such small particles. However, it is reached faster (a shorter relaxation length) in the higher $\eta$ case as is seen from comparing Fig. 14 with Fig. 16 and 


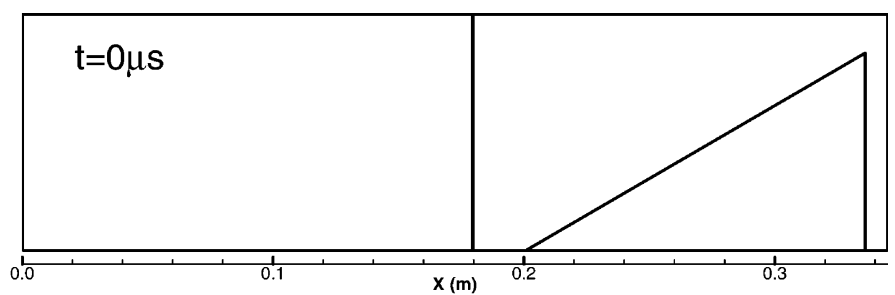

(a)

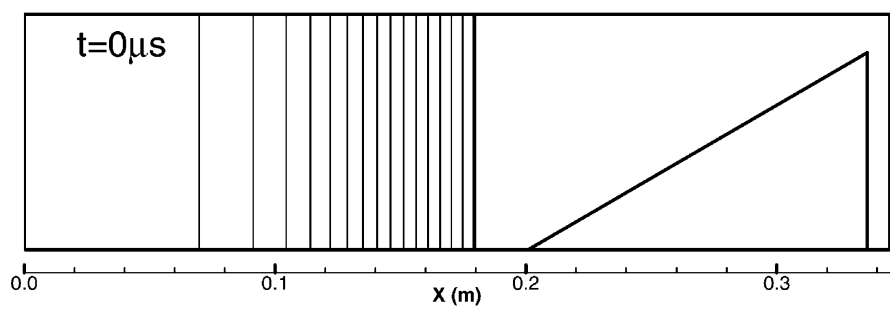

(b)

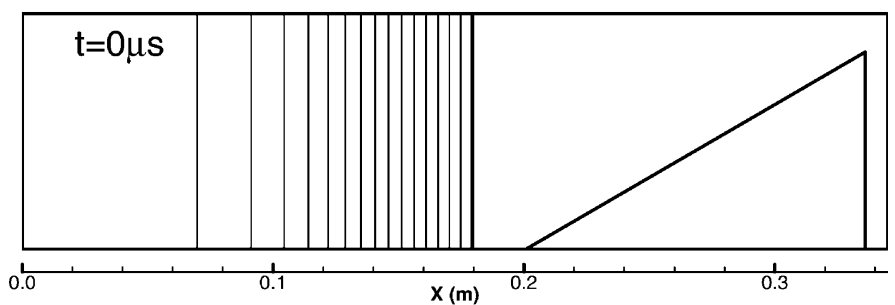

(c)

Fig. 9. Shock wave reflection from a wedge placed inside a suspension. $M_{\mathrm{s}}=1.384, P_{0}=1$ bar, $T_{0}=294 \mathrm{~K}$ and $D_{\mathrm{p}}=10$ $\mu \mathrm{m}$ : (a) pure gas, (b) suspension having a dust loading equal 0.1 and (c) dust loading equal $1.0, \mathrm{t}=0 \mu \mathrm{s}$.

from Table 1. Once the dust particle diameter is increased to $5 \mu \mathrm{m}$, or higher, the observed wave pattern in the solid phase is completely different from that seen in the isopycnics map of the gaseous phase; compare Fig. 16 with Figs. 7c and 8c. Here no shock or compression waves are visible. However, as observed in the $\eta=0.1$ case, a narrow zone of high dust concentration is observed near the wedge surface (see Fig. 16). In order to clearly demonstrate the existence of steep gradients in the dust spatial density, which take place near the wedge surface, results obtained for $\eta=1.0$ and $D_{\mathrm{p}}=5 \mu \mathrm{m}$ are shown in Fig. 17. In this figure, where $Y$ is the coordinate normal to the wedge surface, results are shown for $t=300 \mu$ s and for three different location along the wedge. The coordinate $X$ is along the wedge, starting from the leading edge. It is clearly visible from Fig. 17 that close to the wedge surface there exists a thin layer (about $4 \mathrm{~mm}$ thick) in which a fast reduction in the dust spatial density takes place as function of the distance $(Y)$ from the wedge surface.

The results shown so far were obtained using a sticking (no-rebound) boundary condition for the dust particles impinging at all solid surfaces. Naturally we would like to estimate the effect of this boundary condition on the reflection wave pattern. An alternate (and very different) 


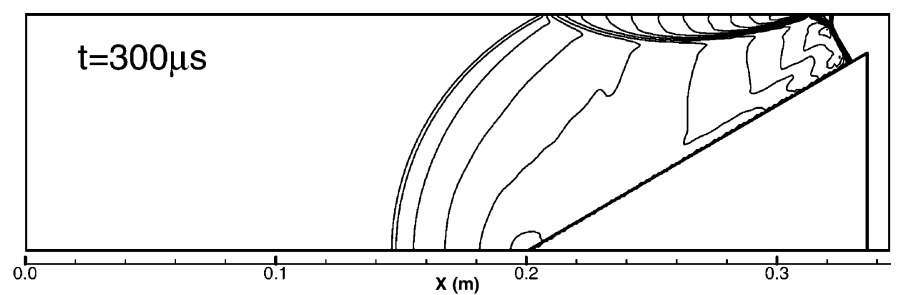

(a)

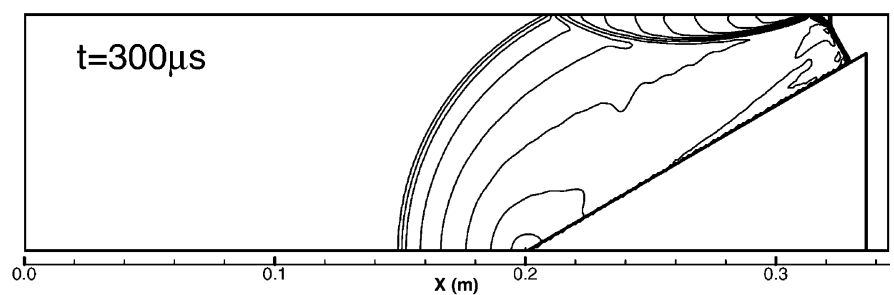

(b)

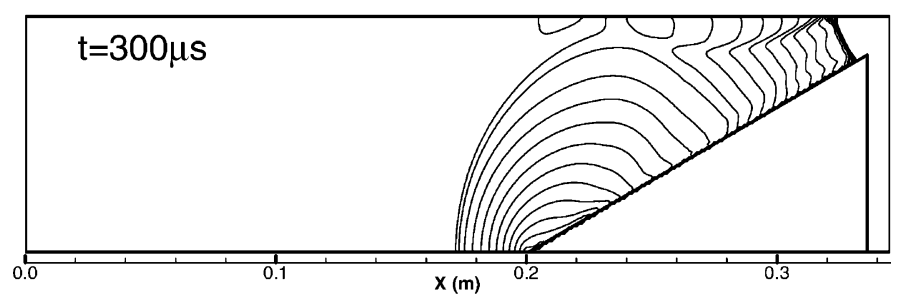

(c)

Fig. 10. Shock wave reflection from a wedge placed inside a suspension. $M_{\mathrm{s}}=1.384, P_{0}=1$ bar, $T_{0}=294 \mathrm{~K}$ and $D_{\mathrm{p}}=10 \mu \mathrm{m}$ : (a) pure gas, (b) suspension having a dust loading equal 0.1 and (c) dust loading equal $1.0, t=300 \mu \mathrm{s}$.

boundary condition is of elastic reflection of particles arriving at the wall. We have in fact repeated some of the previously presented cases with this boundary condition, observing virtually no difference in the reflected wave pattern. For a specific instance of comparison between the two boundary conditions we refer the reader to our previous study of shock wave interactions in dusty gas (e.g., Fig. 4a and c in Igra et al., 2002). Another point in this regard is the apparent accumulation of particles near the wedge surface as seen in Figs. 14 and 16. Since our treatment of the boundary condition is only via one of the two extreme alternatives, and we do not know a more physically correct boundary condition, we suggest that particle accumulation near the wedge surface be taken as possible, but uncertain, feature of the shock reflection flow.

\subsection{Equilibrium suspension (Pseudo-Gas)}

A suspension at a state of equilibrium could be represented as a pseudo-gas having a specific heats ratio $\left(\gamma=C_{p} / C_{v}\right)$ which depends solely on the dust loading ratio, $\eta$, and the ratio of solid-togas specific heats, $\delta=C_{m} / C_{p}$. Rudinger (1980) showed that $\gamma$ for such a pseudo-gas is given by: 


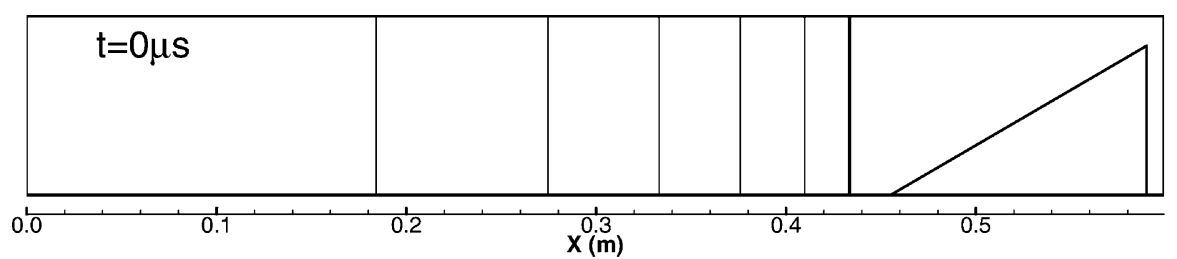

(a)

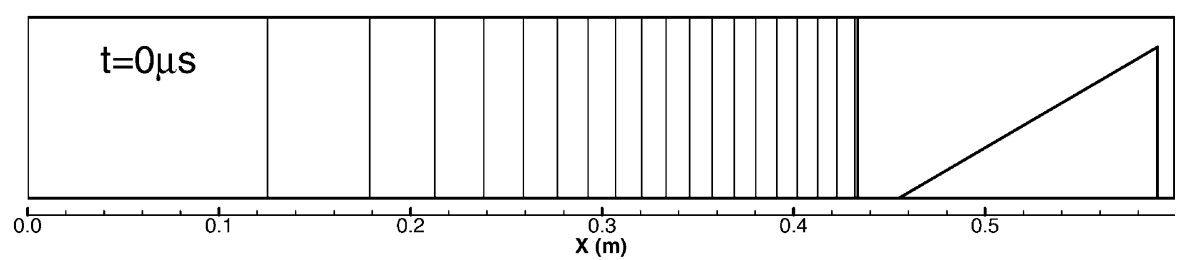

(b)

Fig. 11. Shock wave reflection from a wedge placed inside a suspension. $M_{\mathrm{s}}=1.384, P_{0}=1$ bar, $T_{0}=294 \mathrm{~K}$ and $D_{\mathrm{p}}=20 \mu \mathrm{m}$, (a) a suspension having dust loading equal to 0.1 and (b) dust loading equal $1.0, t=0 \mu \mathrm{s}$.

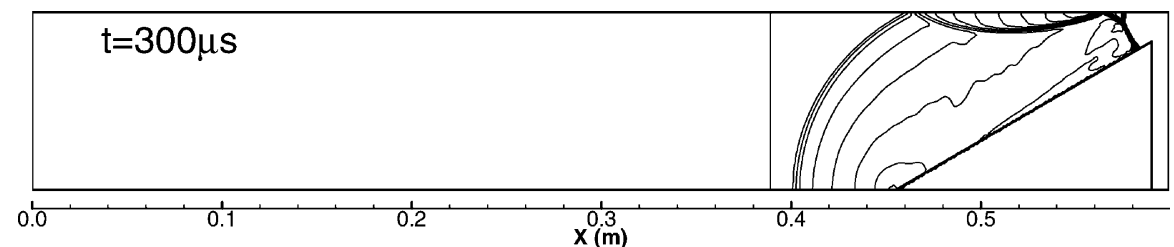

(a)

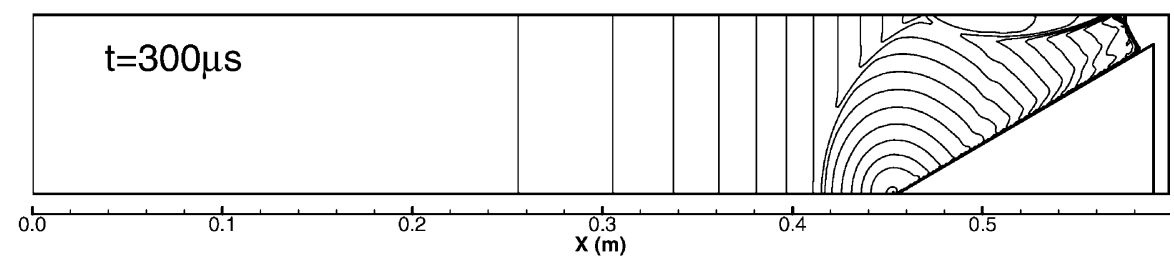

(b)

Fig. 12. Shock wave reflection from a wedge placed inside a suspension. $M_{\mathrm{s}}=1.384, P_{0}=1$ bar, $T_{0}=294 \mathrm{~K}$ and $D_{\mathrm{p}}=20 \mu \mathrm{m}$, (a) a suspension having dust loading equal to 0.1 and (b) dust loading equal $1.0, t=300 \mu \mathrm{s}$.

$$
\gamma_{\text {p.g. }}=\gamma(1+\eta \delta / \gamma) /(1+\eta \delta)
$$

The gas constant $\bar{R}$ in the pseudo-gas at equilibrium state is $\bar{R}=R /(1+\eta)$ where $R$ is the gas constant of the pure gas (air in the present case). As shown in Falcovitz and Igra (2000), at late time all suspension properties behind a traveling shock wave will approach the values obtained by replacing the two-phase suspension with a pure gas having $\gamma=\gamma_{\text {p.g. }}$ and using this value of $\gamma$ 

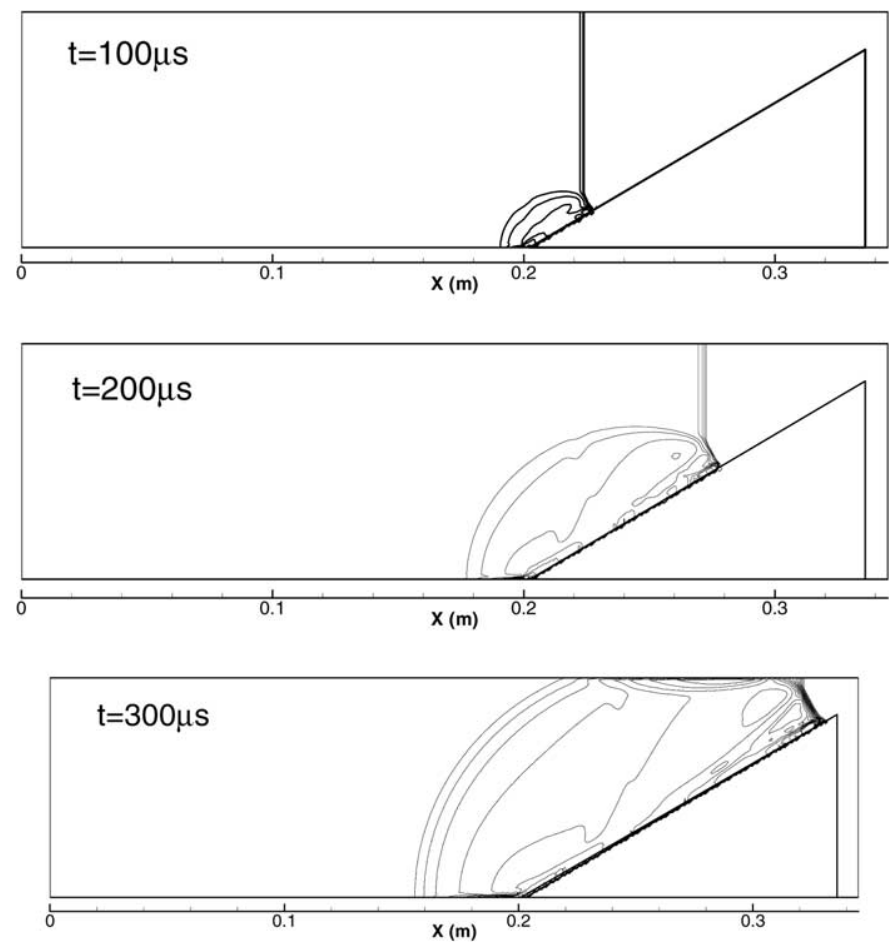

Fig. 13. Isopycnics in the solid phase of the suspension. Initial conditions are: $M_{\mathrm{s}}=1.384, P_{0}=1$ bar, $T_{0}=294 \mathrm{~K}$, $\eta=0.1$ and $D_{\mathrm{p}}=1 \mu \mathrm{m}$.

in the Rankine-Hoguniot shock relations. In Fig. 18 results obtained for shock wave reflection from the considered wedge $\left(\theta=30.5^{\circ}\right)$ are shown during the reflection process and for three different cases. The case labeled (a) indicates shock reflection in a pure gas (air, $\gamma=1.4$ ). Case (b) shows the reflection in the considered suspension; these results are identical to those shown before. Case (c) shows results obtained for a suspension at equilibrium when it is taken as a pure gas (having the appropriate $\gamma_{\text {p.g. }}$ ). In such a case the pressure jump across the traveling shock wave is the jump from the pre-shock pressure level $P_{0}$, to the pressure prevailing in the suspension behind the incident shock, where an equilibrium state is reached, i.e., at the end of the relaxation zone. As shown subsequently, this equilibrium pressure in the suspension is significantly higher than the frozen pressure jump across the air-shock and therefore it corresponds to a higher shock wave Mach number (with respect to the pseudo-gas). We reiterate that in the dusty suspension the incident shock wave Mach number is defined with respect to the speed of sound in the pure gas. It is apparent from Fig. 18 that the reflection wave pattern obtained for the suspension (case b) bears a similarity to the wave pattern seen in the eventually reached equilibrium state (case c). This is especially so at late times $(t=300 \mu \mathrm{s})$, where the observed reflection wave pattern in the suspension (case b) is very different from the pattern seen in a similar pure gas (case a) but it has a similar geometry to that of the equilibrium case of Fig. 18c. As already mentioned, in the case of a low dust loading and small solid particles 

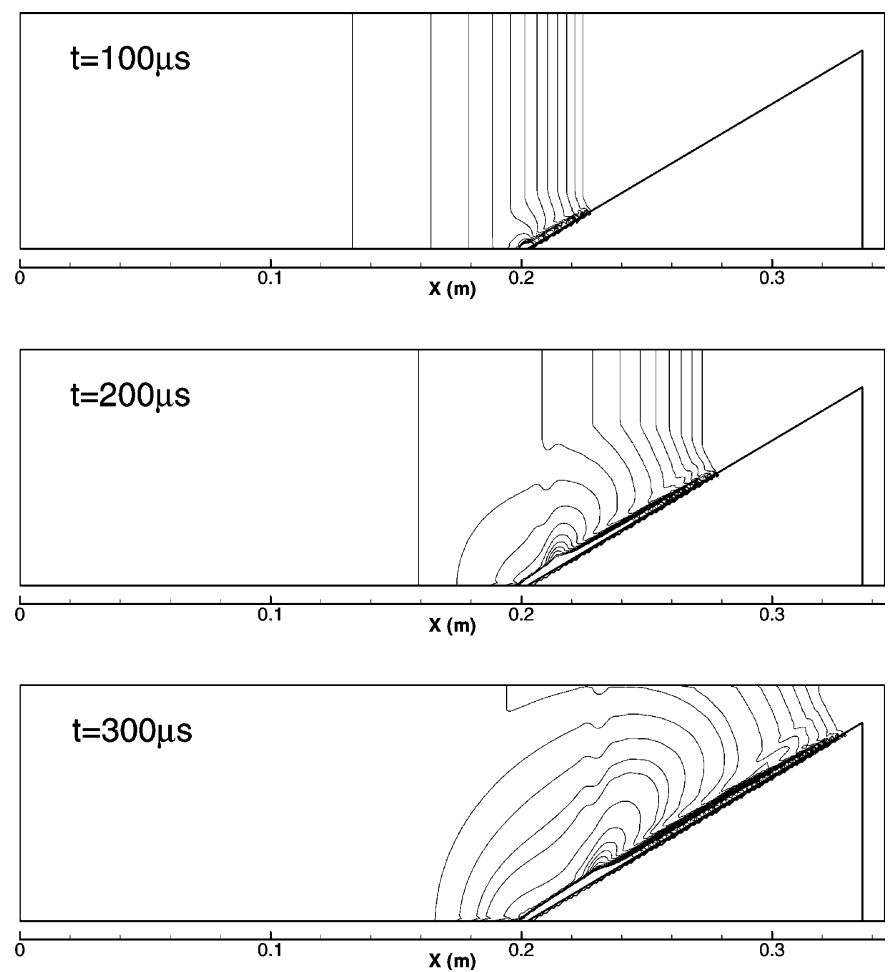

Fig. 14. Isopycnics in the solid phase of the suspension. Initial conditions are: $M_{\mathrm{s}}=1.384, P_{0}=1$ bar, $T_{0}=294 \mathrm{~K}$, $\eta=0.1$ and $D_{\mathrm{p}}=5 \mu \mathrm{m}$.

(where $\eta=0.1$ and $D_{\mathrm{p}}=1 \mu \mathrm{m}$ ) the suspension reaches equilibrium relatively quickly and the observed reflection wave pattern is similar to that seen in the corresponding pure gas case. Results obtained for such conditions, at $t=300 \mu$ s are shown in Fig. 19 (similar agreement between pure gas, suspension and equilibrium cases are obtained at earlier times as well and therefore are not shown here).

\subsection{Pressure distribution on the wedge surface}

So far the effects of the dust presence were shown only with respect to the resulting wave pattern developed over the wedge. The effect of the dust presence on the prevailing pressure over the wedge surface is shown in Fig. 20. Fig. 20a and c show pressure histories at a distance of 23 $\mathrm{mm}$ from the wedge leading edge while those shown in Fig. 20b and d are for a distance of $143 \mathrm{~mm}$ from the wedge leading edge. Fig. 20a and $\mathrm{b}$ are for $\eta=1$ and those shown in Fig. 20c and d are for $\eta=0.1$. The following facts are clearly seen in Fig. 20: Increasing the dust loading ratio $\eta$, significantly increases the suspension pressure and the time past until an equilibrium state is reached. For the case $\eta=0.1$ (Fig. 20c), the post-shock suspension equilibrium pressure is about 3.1 bar and for suspension composed of $5 \mu \mathrm{m}$ particles equilibrium is reached at about $250 \mu$ s after the passage of the incident shock wave. When $\eta$ is increased to $\eta=1.0$ (Fig. 20a) the post-shock 

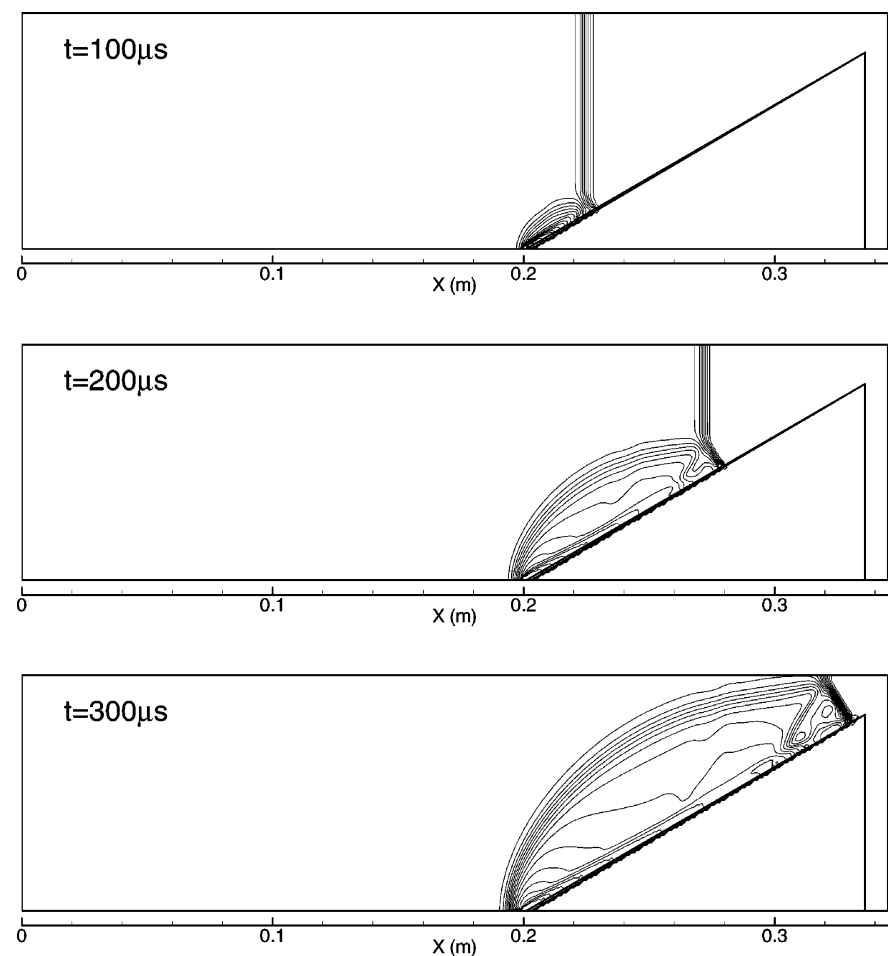

Fig. 15. Isopycnics in the solid phase of the suspension. Initial conditions are: $M_{\mathrm{s}}=1.384, P_{0}=1$ bar, $T_{0}=294 \mathrm{~K}$, $\eta=1$ and $D_{\mathrm{p}}=1 \mu \mathrm{m}$.

suspension pressure exceeds 10 bars. It should be noted that in a similar (same $M_{\mathrm{s}}$ ) pure gas case the post-shock pressure acting on the wedge is only 2.8 bars. As could be expected, the larger the particle diameter, the longer it takes for an equilibrium state to be established. In Fig. 20c the 5 $\mu \mathrm{m}$ particle-suspension reaches equilibrium state within the investigated time; it takes longer time for suspensions composed of larger particles. However, it is clear from Fig. 20a and c that the three shown suspensions (with $D_{\mathrm{p}}=5,10$, and/or $20 \mu \mathrm{m}$ ), indicate approach to the same equilibrium pressure. This fact was also observed elsewhere (e.g. in Hoglund, 1962; Panton, 1968; Rudinger, 1980 and in Igra and Ben-Dor, 1988). Results shown in Fig. 20b and d show pressure histories at a location close to the wedge rear end. Obviously it takes longer time for the incident shock wave to reach this position, as shown in these figures, and for the short time (covered in these figures) after the passage of the incident shock at this location no equilibrium state could be reached. However, the significant increase in the post-shock pressure observed in suspensions relative to a similar pure gas case is clearly evident in Fig. 20b and d. It should be noted that the incident shock wave was specified by the same frozen (leading) air shock Mach number $\left(M_{\mathrm{s}}=1.3843\right)$ for all cases. This choice was motivated by seeking a comparative study of the effects of dust loading ratio on the wave reflection pattern. However, it also resulted in (equilibrium) post-shock pressures that increased significantly with the increase in the dust loading ratio. This effect is apparent in the wedge surface pressures shown in Fig. 20a-d. In other words, 

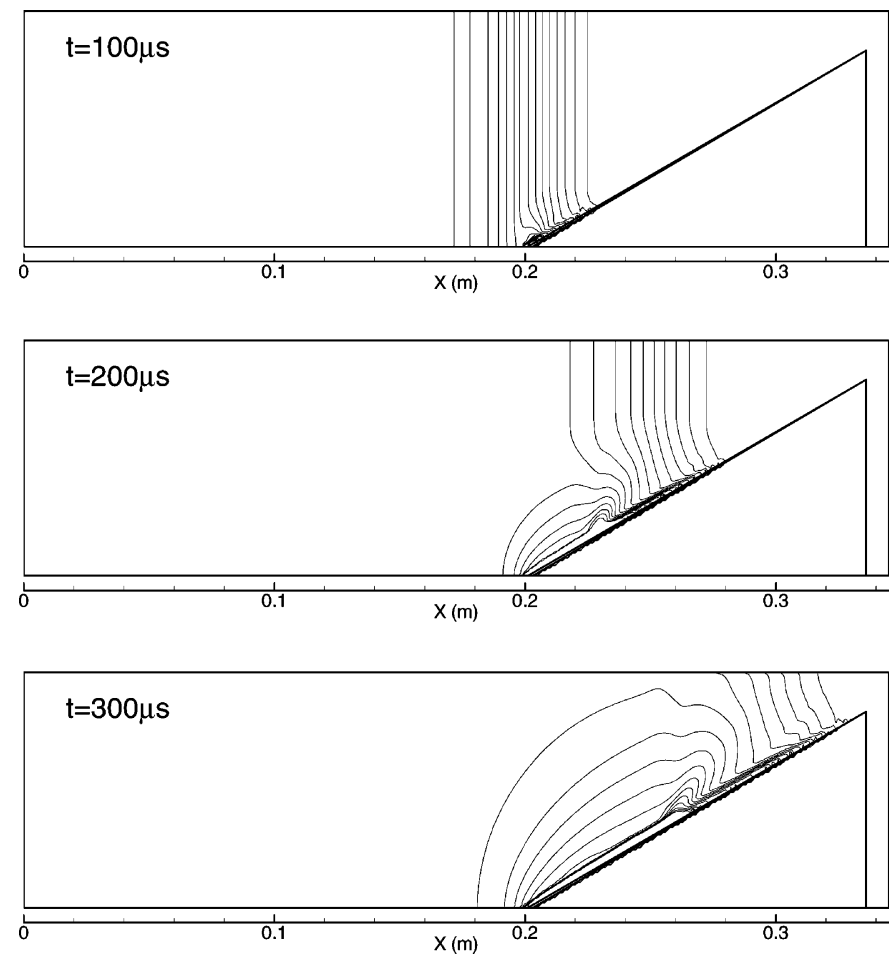

Fig. 16. Isopycnics in the solid phase of the suspension. Initial conditions are: $M_{\mathrm{s}}=1.384, P_{0}=1$ bar, $T_{0}=294 \mathrm{~K}$, $\eta=1$ and $D_{\mathrm{p}}=5 \mu \mathrm{m}$.

with respect to the wedge surface pressure, the comparative cases presented here demonstrate the combined effects of the increased dust loading ratio, along with the modified reflection shock flow field.

The effect of the dust presence, and the effect played by the dust size on the approach to equilibrium could also be seen in Figs. 21-23 where the pressure distribution along the wedge surface is shown for different times and different dust loadings. In Fig. 21 results obtain for the smallest particles $\left(D_{\mathrm{p}}=1 \mu \mathrm{m}\right)$ are shown along with those obtained for an equilibrium case. In Figs. 21-23 the location $L=0$ indicates the wedge leading edge. As could be expected, Fig. 21 shows that for suspensions composed of very small particles the approach to equilibrium is very fast. Throughout most of the wedge surface there is very good agreement between numerical results obtained for the pressure distribution along the wedge in the considered suspension and the equilibrium prediction obtained while expressing the suspension as an equivalent pure gas having the appropriate values for $\gamma_{\text {p.g. }}$ and for $\bar{R}$. It is also clear from Fig. 21 that the dust presence causes significant increase in the pressures prevailing over the wedge surface, relative to a similar pure gas case. As could be expected increasing the dust loading ratio in the suspension causes a significant increase in the prevailing pressure. Results shown in Figs. 22 and 23 indicate the approach to equilibrium when the suspension's particles have larger diameters. It is apparent from Fig. 22, where results for the diluted suspension $(\eta=0.1)$ are shown, that at early times $(t \leqslant 200 \mu \mathrm{s})$ the 

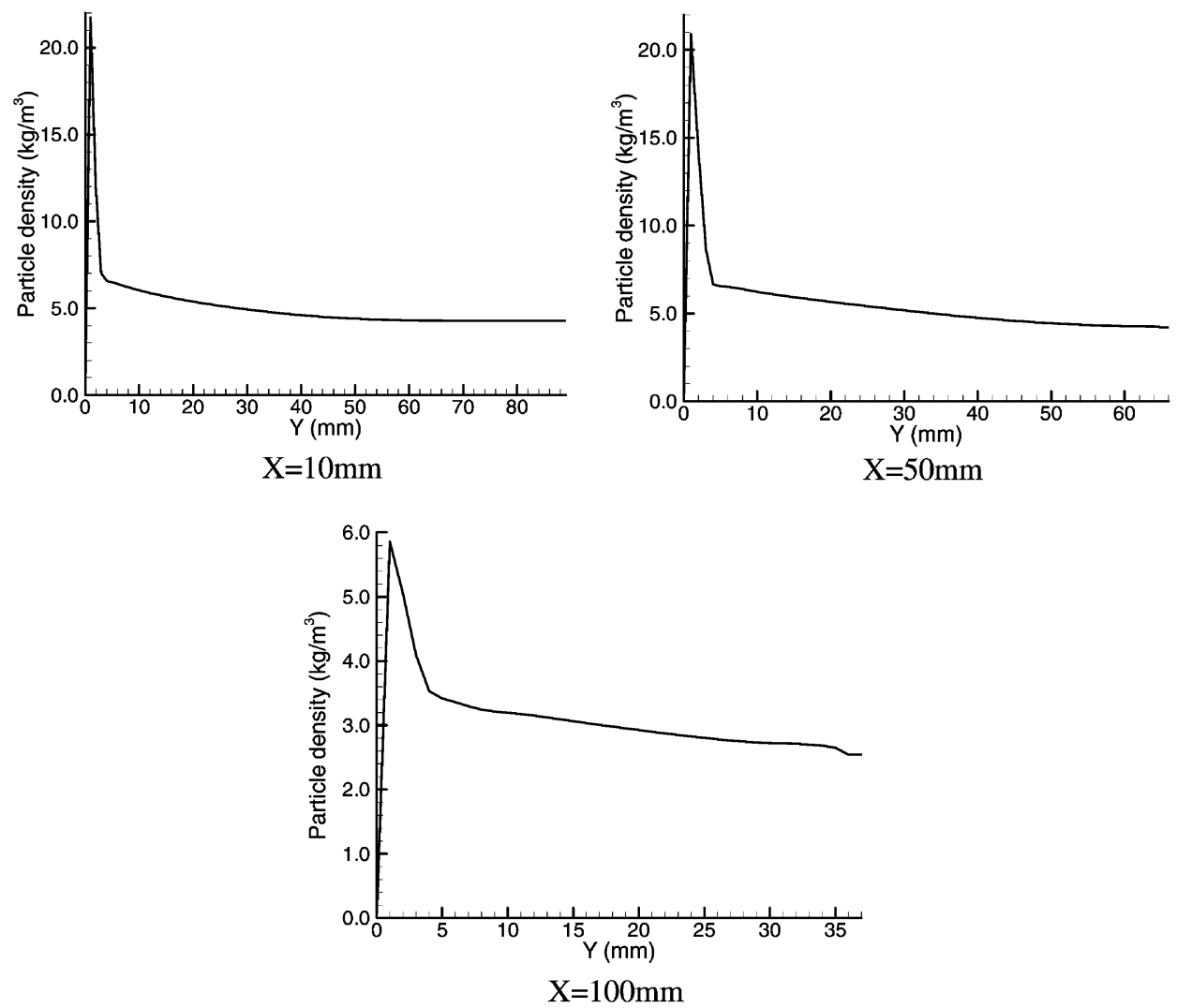

Fig. 17. Spatial dust density distribution vertical to the wedge surface for $\eta=1, D_{\mathrm{p}}=5 \mu \mathrm{m}$ at $t=300 \mu$ s. Initial conditions are: $M_{\mathrm{s}}=1.384, P_{0}=1$ bar and $T_{0}=294 \mathrm{~K}$.

pressures for $D_{\mathrm{p}} \geqslant 5 \mu \mathrm{m}$ are between the equilibrium limit (which is very close to the $D_{\mathrm{p}}=1 \mu \mathrm{m}$ line as shown previously) and the pure gas pressure (which is identical to a frozen flow case). With progressing time the lines representing pressure distribution for particles of different sizes moves towards the equilibrium limit. For $t=300 \mu$ s the suspension composed of particles having a diameter of $5 \mu \mathrm{m}$ reaches equilibrium conditions close to the wedge leading edge but not further down the wedge. As could be expected, the suspension composed of the largest particles $\left(D_{\mathrm{p}}=20\right.$ $\mu \mathrm{m})$ is still far from reaching the equilibrium pressure due to the relative high inertia of the solid particles. Results obtained for a denser suspension $(\eta=1)$ are shown in Fig. 23. Now all results obtained for suspensions in which $D_{\mathrm{p}} \geqslant 5 \mu \mathrm{m}$ are far from the equilibrium pressure level. The pressure distribution over the wedge surface in a suspension composed of particles with $D_{\mathrm{p}}=5$ $\mu \mathrm{m}$ are close to that obtained for $D_{\mathrm{p}}=1 \mu \mathrm{m}$ case but still not there.

In Fig. 23, for the case where $t=300 \mu$ s, a peculiar behavior is observed in the suspension pressure distribution over the wedge (within the region $100 \mathrm{~mm}<L<150 \mathrm{~mm}$ ), for the case where $\eta=1$ and $D_{\mathrm{p}}=1 \mu \mathrm{m}$. The existence of such a pressure variation could be predicted by inspecting the isopycnics shown in Fig. 5c. The observed isopycnics behind the Mach stem in Fig. 5c are 

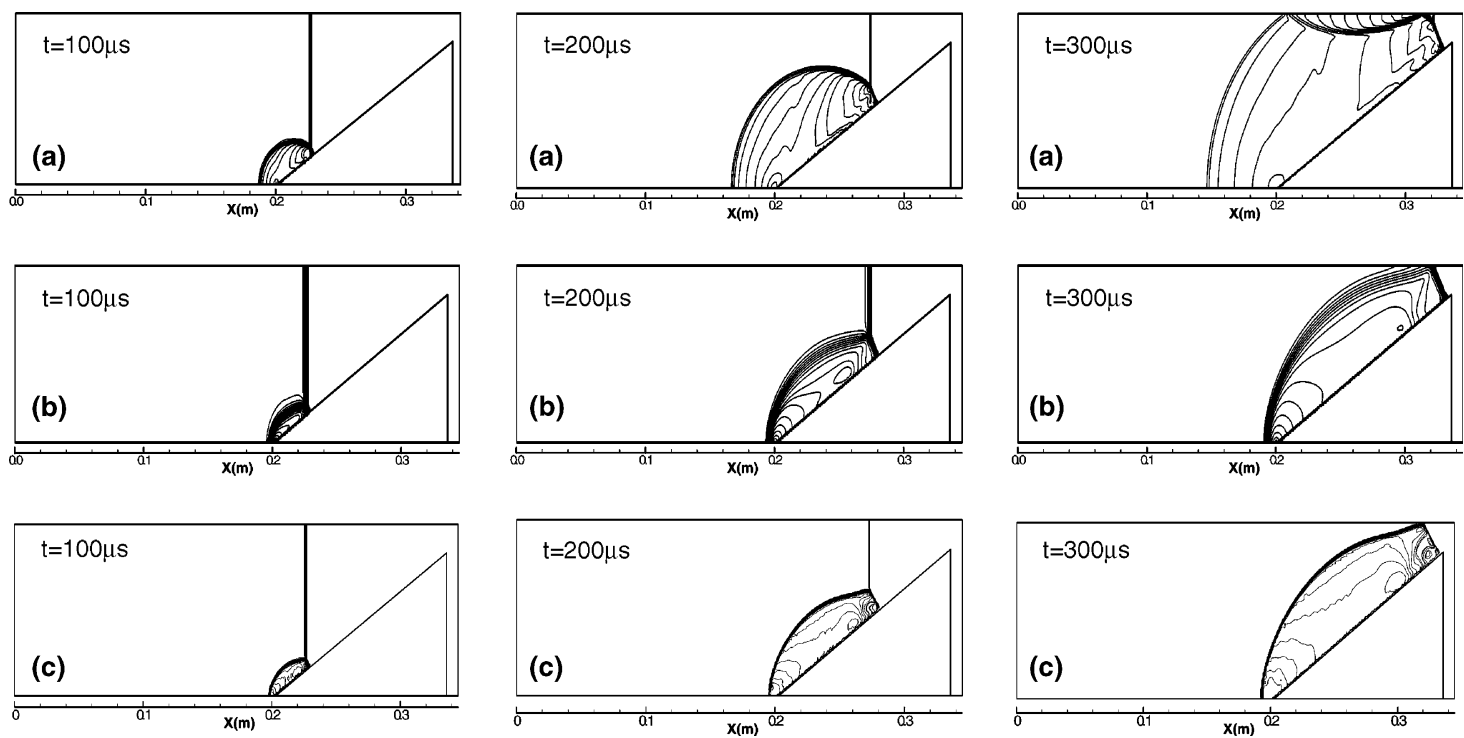

Fig. 18. Shock wave reflection from a wedge. (a) Pure gas, $P / P_{0}=2.069, M_{\mathrm{s}}=1.3843$. (b) Frozen air shock, $P / P_{0}=2.069, M_{\mathrm{s}}=1.3843, \eta=1.0, D_{\mathrm{p}}=1 \mu \mathrm{m}$. (c) Equilibrium gas shock, $P / P_{0}=4.87, M_{\mathrm{s}}=2.132, \gamma=1.167$, $\rho_{0}=2.37 \mathrm{~kg} / \mathrm{m}^{3}$.

different from those seen in Figs. 8c, 10c and 12c where they follow a fairly regular (parallel) pattern. For verifying this pressure distribution, we repeated the computation with half the time step $(\mathrm{CFL}=0.25)$ and obtained virtually identical results.

With respect to shock wave reflection from a wedge, a frequently asked question is: How would the dust presence affect the reflection pattern? Or, more specifically, would the transition from regular to Mach reflection depend upon the magnitude of the dust loading ratio in the suspension?

In two previously quoted numerical studies (Kim and Chang, 1991, Ben-Dor et al., 2001) it was suggested that Mach-to-regular transitions are affected by the presence of dust in the suspension. For example Fig. 13 in Ben-Dor et al. (2001), which is shown here as Fig. 24, clearly suggests that in the case of an incident shock wave propagating at $M_{\mathrm{s}}=1.5$ in pure air, a regular reflection from a wedge will take place if the wedge deflecting angle is equal to, or larger than $50.25^{\circ}$. For smaller angles a Mach reflection takes place. As is evident from Fig. 24, the presence of a dusty suspension around the wedge results in a lower transition angle for the same pure gas incident shock wave. Furthermore, the transition angle is found to decrease as the dust loading is increased.

We do not object to these observations per se, but to their interpretation as indicating a "transition envelope" between regular and Mach reflection. In the previously mentioned studies, the two-dimensional flow domain in the $(x, y)$ plane consists of two parts, say $[x<0, y>0]$ and $[x>0, y>0]$, with the wedge leading edge located at $(x, y)=(0,0)$. The dusty suspension occupies solely the space above the wedge, whereas the rectangular part $[x<0, y>0]$ is filled with pure gas. Initially, the incident shock wave is positioned in the pure gas and propagates towards the wedge, so that its reflection commences upon the arrival of the shock front at the line $x=0$. 


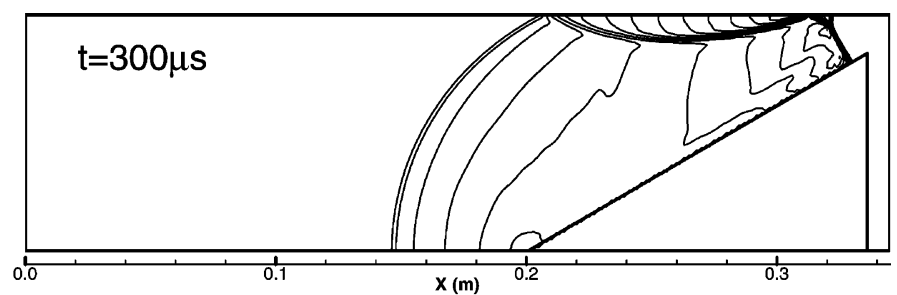

(a)

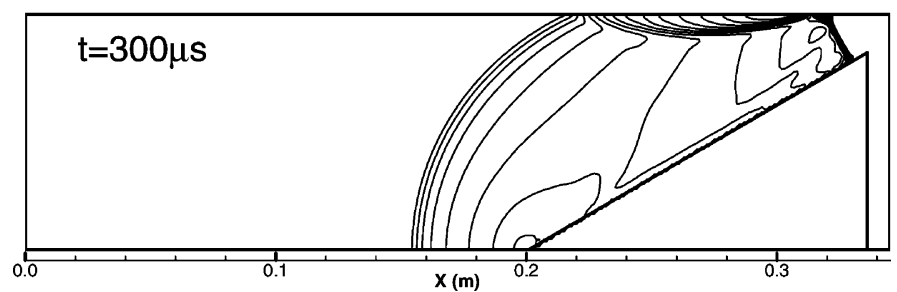

(b)

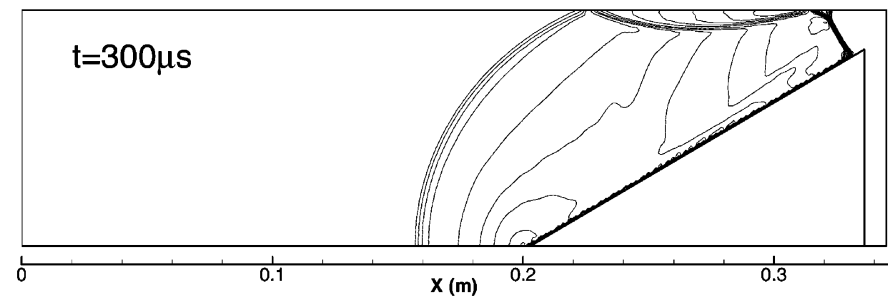

(c)

Fig. 19. Shock wave reflection from a wedge. (a) Pure gas, $P / P_{0}=2.069, M_{\mathrm{s}}=1.3843$. (b) Frozen air shock, $P / P_{0}=2.069, M_{\mathrm{s}}=1.3843, \eta=0.1, D_{\mathrm{p}}=1 \mu \mathrm{m}$. (c) Equilibrium gas shock, $P / P_{0}=2.3654, M_{\mathrm{s}}=1.497, \gamma=1.351$, $\rho_{0}=1.305 \mathrm{~kg} / \mathrm{m}^{3}$.

However, we note that two unrelated processes start at that moment:

(i) The incident shock is reflected from the wedge surface and;

(ii) The interaction between the pure gas shock and the suspension along the entire interface $[x=0, y>0]$, results in a transmitted shock wave propagating into the suspension, and a reflected shock propagating back into the pure gas.

The transmitted shock evolves over time its intensity being gradually attenuated from that of the incident shock to a lower level as it propagates into the suspension. Thus, the intensity of the incident shock arriving at the wedge surface decreases with the distance from the leading edge, and its reflection pattern cannot be characterized by a single-valued data.

Obviously, the reason for all this is the particular configuration chosen by Kim and Chang (1991), and by Ben-Dor et al. (2001), where the incident shock propagates initially in pure gas, and where the suspension commences at the wedge leading edge. In our configuration, by 

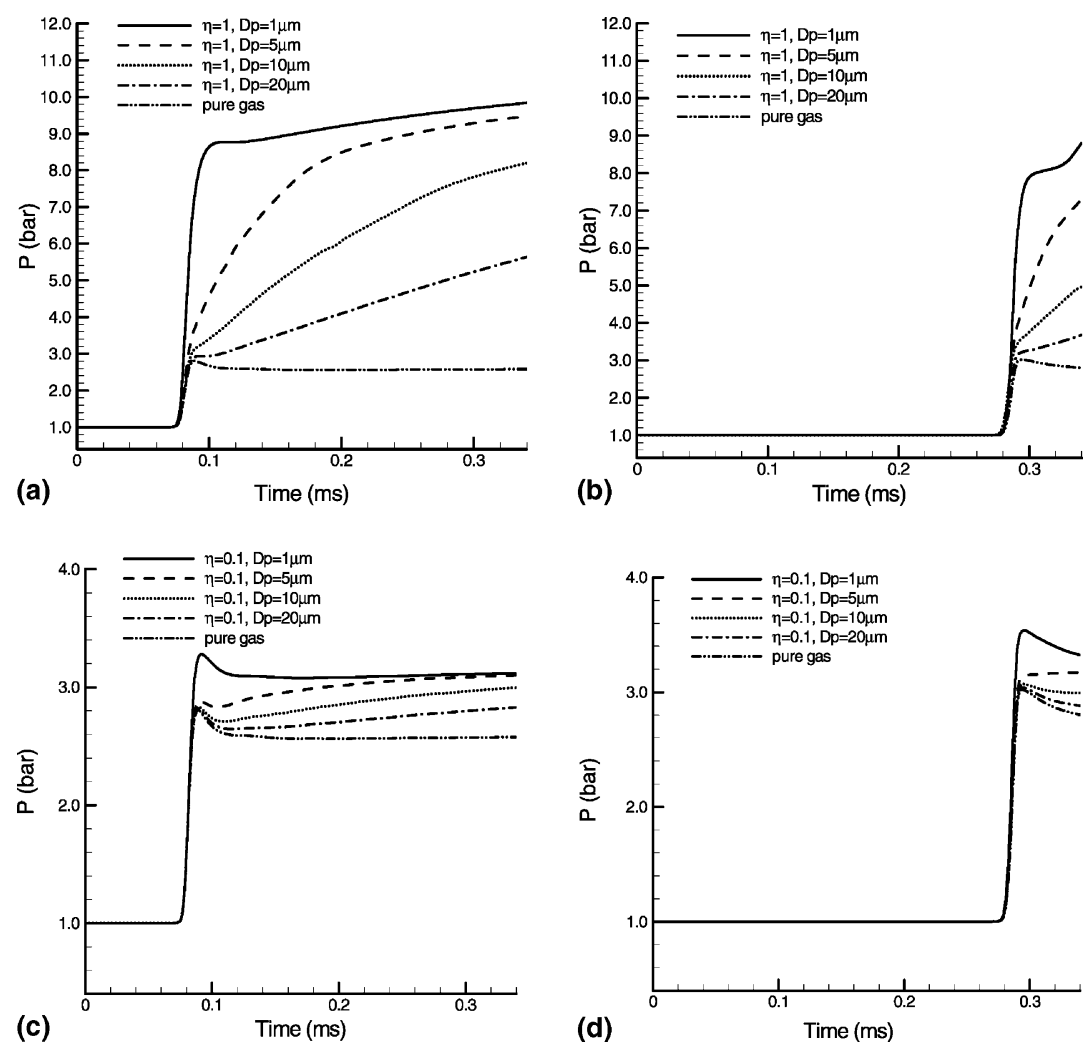

Fig. 20. Pressure histories at different positions along the wedge surface.

contrast, the suspension occupies the entire space, and the incident shock is fully accommodated to the suspension prior to its arrival at the wedge leading edge (in other words, the flow profile following the shock front is stationary in a coordinate system moving with the shock front). A series of computations conducted with this configuration is presented in Fig. 25, with the incident shock front moving at Mach number $M_{\mathrm{s}}=1.5$ (with respect to the pure gas speed of sound).

As is apparent from the wave patterns shown in Fig. 25, the transition from regular to Mach reflection is virtually independent of the dust loading ratio. This result can be explained by the following reasoning. The relaxation process of dust-gas accommodation behind a shock front takes place at a finite rate, and therefore requires a finite distance ("relaxation length") to achieve thermal and kinematic equilibrium between the solid particles and the gas. Near the front, where the accommodation is just beginning, the gas flow is nearly "frozen", meaning that it is almost identical to that of the corresponding pure gas case. Extending this reasoning to the familiar 3shock pattern of a Mach reflection, the "frozen" reflected shock pattern in the dusty gas case is nearly identical to that obtained in the corresponding pure gas shock reflection, provided the spatial extent of that flow (e.g., length of Mach stem) is much smaller than the relaxation length. Now, this is indeed the case when an incipient Mach reflection is considered, since then the length 

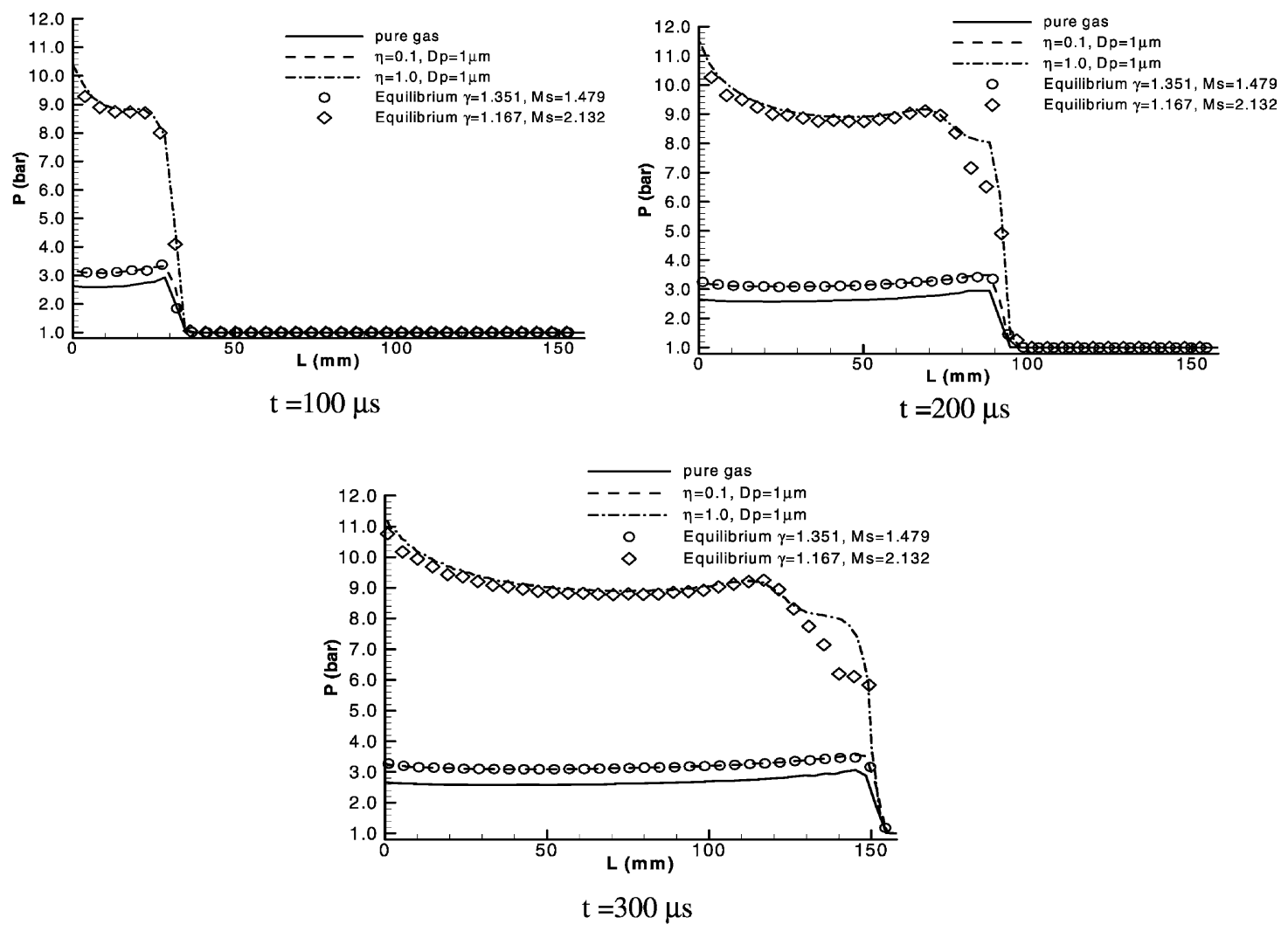

Fig. 21. Pressure distribution along the wedge surface for suspensions composed of small dust particles, $D_{\mathrm{p}}=1 \mu \mathrm{m}$.

of the Mach stem is comparable to the mesh size, and the suspension relaxation length is generally much larger than the mesh size.

\section{Summary and conclusions}

The reflection process of a traveling shock wave from a wedge, placed in a suspension, is studied numerically. For evaluating the effects associated with changes in the dust mass loading in the suspension and in the diameter of the particles composing the solid phase the following cases were investigated: A dilute suspension, in which the dust mass load ratio was 0.1 , and a dense suspension in which $\eta=1$. For each of these suspension four different dust particle diameter were tested, $D_{\mathrm{p}}=1,5,10$ and $20 \mu \mathrm{m}$. The main findings are:

- The reflection wave pattern obtained for a dilute suspension composed of very small solid particles is similar to that observed in a similar pure gas case. 

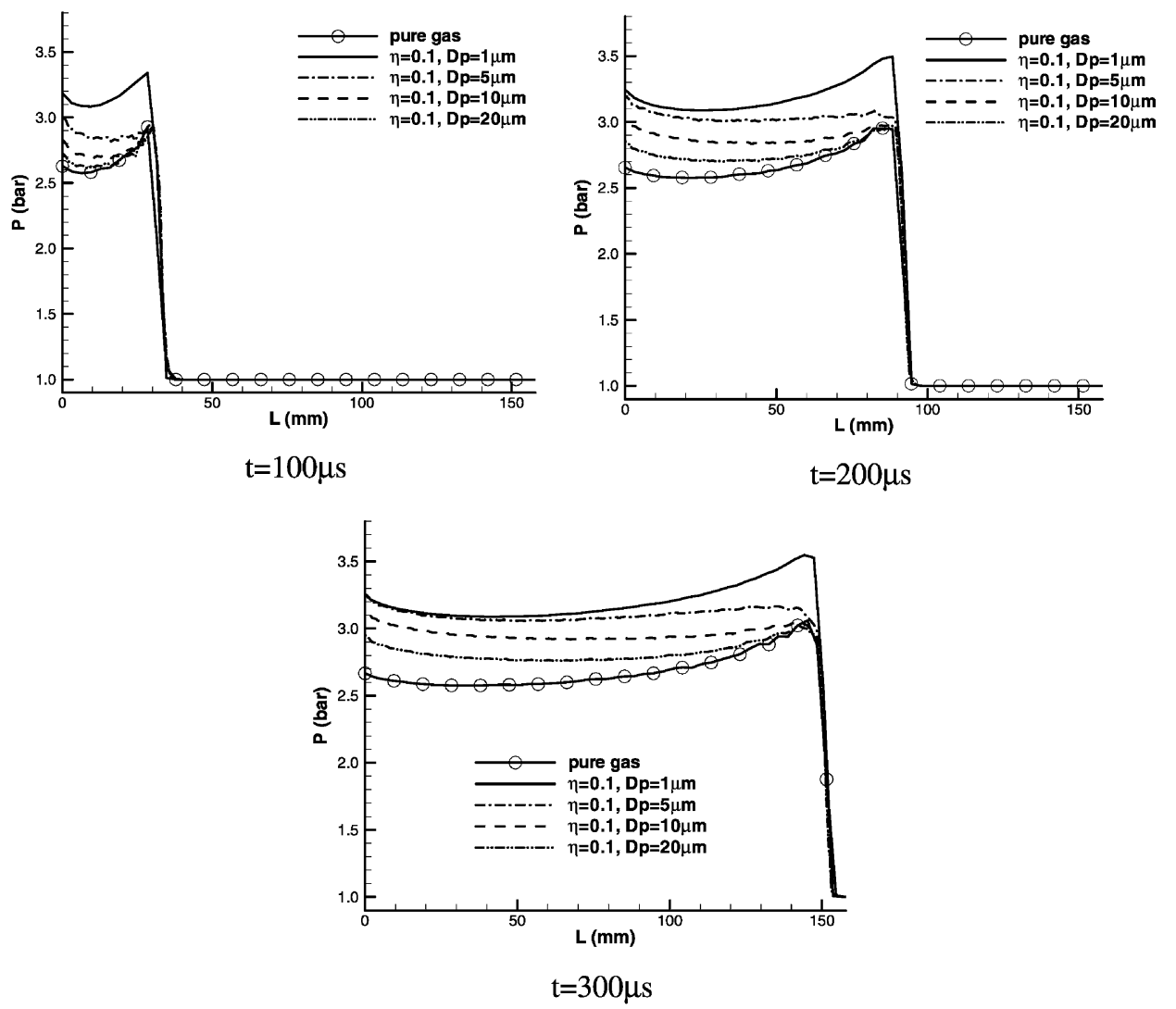

Fig. 22. Pressure distributions along the wedge surface for $\eta=0.1$. For initial conditions see Fig. 18b.

- In suspensions composed of very small solid particles, $D_{\mathrm{p}} \leqslant 1 \mu \mathrm{m}$, the wave pattern exhibited in the gaseous phase isopycnics is similar to that observed in the solid phase isopycnics. This is not surprising since very small particles react quickly to changes in the carrying gas.

- Unlike the dilute suspension, for a dense suspension with particles whose diameter is not too small $\left(D_{\mathrm{p}} \geqslant 1 \mu \mathrm{m}\right)$, all shock waves, except the Mach stem, decay quickly to smooth, continuous waves of compression. In such suspensions the reflected wave pattern observed in the solid phase isopycnics bears no similarity to the respective gaseous phase isopycnics.

- The presence of the solid phase (dust) causes significant increase in the pressure acting on the wedge surface, in comparison with a similar pure gas case. The pressure on the wedge surface following the oblique shock reflection is considerably higher than the respective post-incident shock pressure. It is noted that this "pressure amplification" ratio increases with increasing the dust loading ratio.

- For a given dust loading ratio in a suspension, changing the solid particles diameter changes the time needed to reach an equilibrium pressure. The larger the particles, the longer it takes to reach an equilibrium state. However, suspensions of all particle sizes will eventually reach the same equilibrium pressure level, provided that the dust loading is kept constant. 

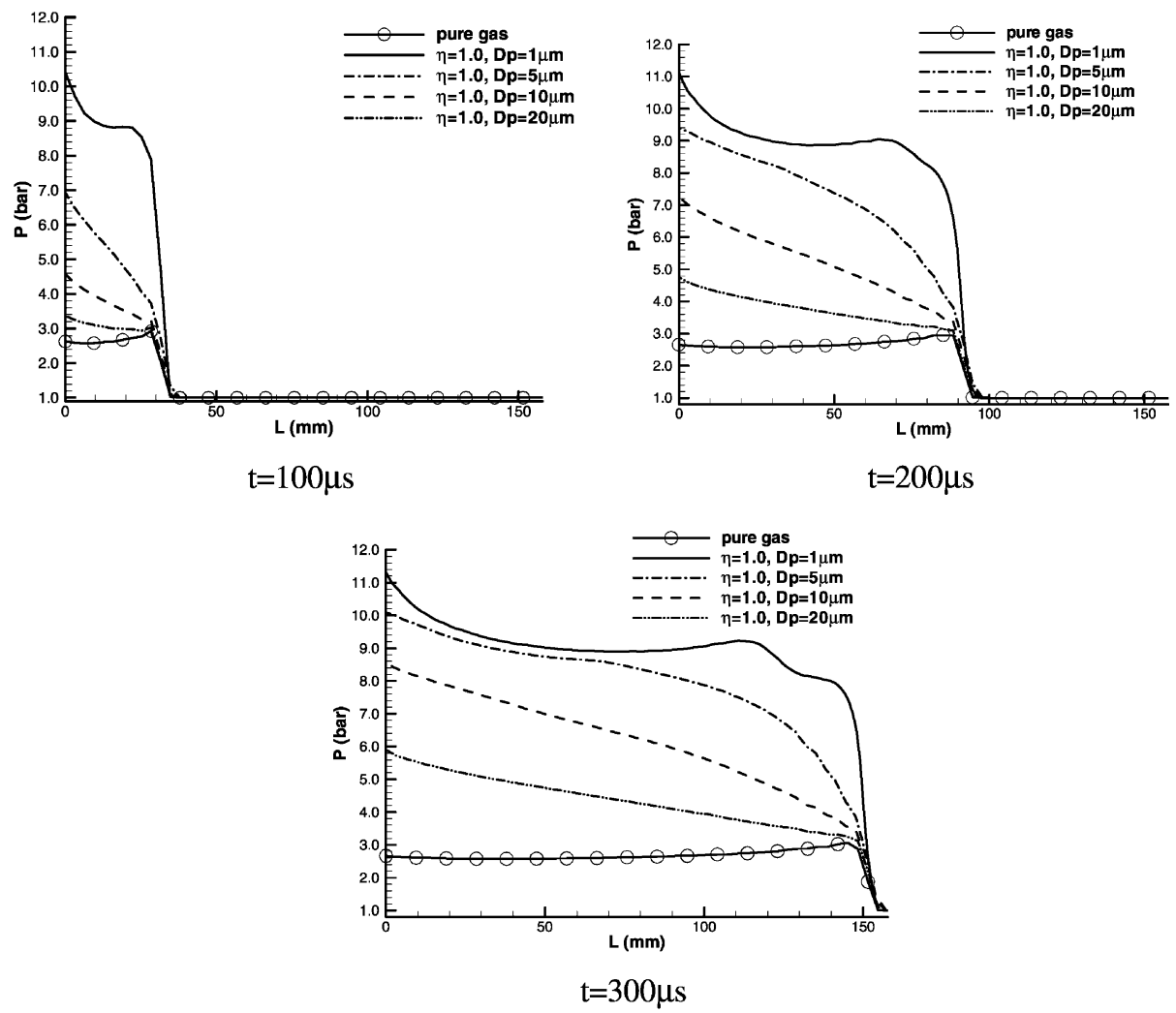

Fig. 23. Pressure distributions along the wedge surface for $\eta=1.0$. For initial conditions see Fig. 18b.

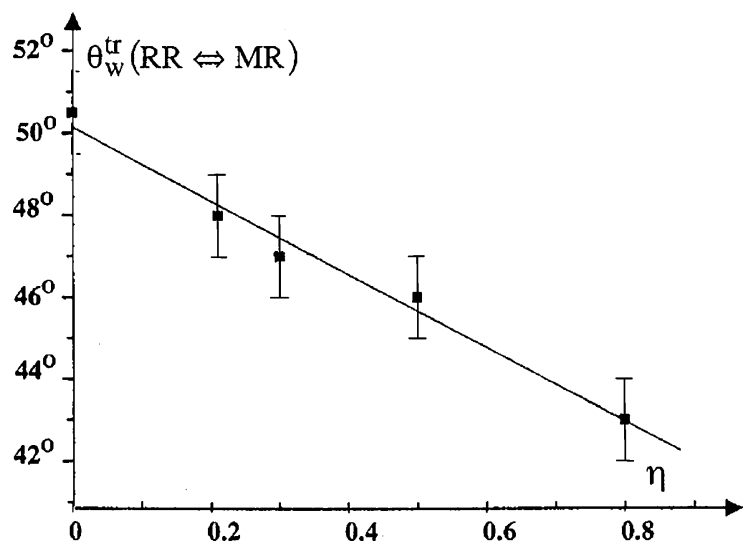

Fig. 24. Variations in the transition angle versus the dust loading ratio in the suspension. $M_{\mathrm{s}}=1.5, D_{\mathrm{p}}=1 \mu \mathrm{m}$. 


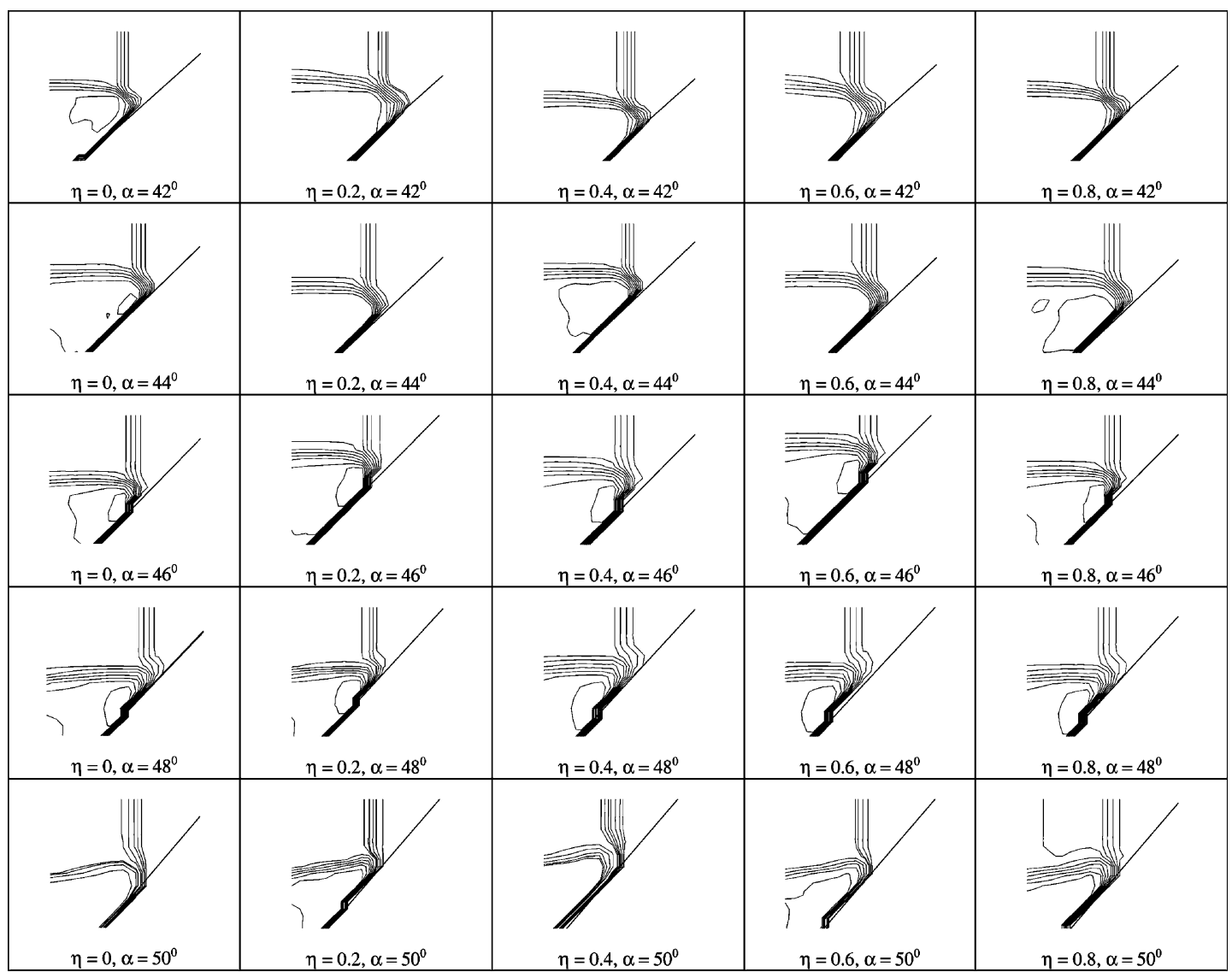

Fig. 25. Reflection pattern obtained for different wedge angles and different dust loadings. In all cases $M_{\mathrm{s}}=1.5$, $D_{\mathrm{p}}=50 \mu \mathrm{m}$.

\section{Acknowledgements}

This work was supported by the German-Israeli Foundation for Research and Development. Their support is acknowledged with thanks.

\section{References}

Ben-Dor, G., 1991. Shock Wave Reflection Phenomena. Springer, New York.

Ben-Dor, G., Igra, O., Wang, L., 2001. Shock wave reflections in dusty-gas suspensions. ASME J. Fluids Eng. 123, 145153.

Crowe, C., Sommerfeld, M., Tsuji, Y., 1998. Multiphase Flows with Droplets and Particles. CRC Press.

Falcovitz, J., Ben-Artzi, M., 1995. Recent developments of the GRP method. JSME Int. J. B. 38, 497-517.

Falcovtiz, J., Alfandary, G., Ben-Dor, G., 1993. Numerical simulation of the head-on reflection of a regular reflection. Int. J. Numer. Methods Fluids 17, 1055-1077. 
Falcovitz, J., Igra, O., 2000. Shock wave structure in dusty gas suspension. In: The 14th Mach Reflection Symposium, October 2000, Sendai, Japan.

Falcovtiz, J., Alfandary, G., Hanoch, G., 1997. A two-dimensional conservation laws scheme for compressible flows with moving boundaries. J. Comput. Phys. 138, 83-102.

Hoglund, R.F., 1962. Recent advances in gas-particle nozzle flow. American Rocket Soc. J. 32, 662-671.

Igra, O., Ben-Dor, G., 1988. Dusty shock waves. Appl. Mech. Rev. 41, 379-437.

Igra, O., Falcovitz, J., Reichenbach, H., Heilig, W., 1996. Experimental and numerical study of the interaction between a planar shock wave and a square cavity. J. Fluid Mech. 313, 105-130.

Igra, O., Wu, X., Falcovitz, J., Meguro, T., Takayama, K., Heilig, W., 2001. Experimental and theoretical study of shock wave propagation through double-bend ducts. J. Fluid Mech. 437, 255-282.

Igra, O., Wu, X., Hu, G.Q., Falcovitz, J., 2002. Shock wave propagation into a dust-gas suspension inside a doublebend conduit. ASME J. Fluids Eng. 124, 483-491.

Kim, S.W., Chang, K.S., 1991. Reflection of shock waves from a compression corner in a particle-laden gas region. Shock Waves 1, 65-73.

Panton, R., Oppenheim, A.K, 1968. Oppenheim. Shock relaxation in a particle-gas mixture with mass transfer between the phases. AIAA J. 6, 2071-2077.

Rudinger, G., 1980. Fundamentals of Gas-Particle Flow. Elsevier, New York. 